\title{
The dynamics of vortex generation in superfluid ${ }^{3} \mathrm{He}-\mathrm{B}$
}

\author{
R. de Graaf • R. Hänninen · T.V. Chagovets • \\ V.B. Eltsov • M. Krusius · R.E. Solntsev
}

Received: 10 Sep, 2008 / Accepted: 11 Sep, 2008

\begin{abstract}
A profound change occurs in the stability of quantized vortices in externally applied flow of superfluid ${ }^{3} \mathrm{He}-\mathrm{B}$ at temperatures $\lesssim 0.6 T_{\mathrm{c}}$, owing to the rapidly decreasing damping in vortex motion with decreasing temperature. At low damping an evolving vortex may become unstable and generate a new independent vortex loop. This single-vortex instability is the generic precursor of turbulence. We investigate the instability with non-invasive NMR measurements on a rotating cylindrical sample in the intermediate temperature regime $(0.3-0.6) T_{\mathrm{c}}$. From comparisons with numerical calculations we interpret that the instability occurs at the container wall, when the vortex end moves along the wall in applied flow.
\end{abstract}

Keywords quantized vortex · vortex formation · vortex instability · vortex dynamics · turbulence $\cdot$ transition to turbulence $\cdot$ mutual friction $\cdot$ rotating flow $\cdot$ vortex remanence

PACS 67.57.Fg · 47.32.-y $\cdot 67.40 . \mathrm{Vs}$

\section{INTRODUCTION}

Turbulence in a rotating fluid is a most frequently encountered phenomenon, ranging from astrophysical and planetary scales to engineering problems. Turbulence in a rotating superfluid has not been extensively investigated, although it is a simpler form of turbulence from that in viscous fluids and might provide a shortcut to new understanding. At temperatures below $2.5 \mathrm{mK}$, uniform rotation is one of the few technically feasible means of generating flow on the global scale. The main reason for studying superfluid ${ }^{3} \mathrm{He}-\mathrm{B}$ at these temperatures is to learn about the influence of a strongly temperature dependent vortex damping [1, or mutual friction dissipation $\alpha(T)$. With

R. de Graaf · R.E. Solntsev · V.B. Eltsov · R. Hänninen · M. Krusius

Low Temperature Laboratory, Helsinki University of Technology, FIN-02015 TKK, Finland

Tel.: +358503442189

Fax: +35894512969

E-mail: rdegraaf@boojum.hut.fi

T.V. Chagovets

Institute of Physics ACSCR, Na Slovance 2, 18221 Prague, Czech Republic 
decreasing temperature a sudden onset of turbulence is seen in applied flow. It is this onset and the mechanisms behind it that are described in this report. An instability of a single vortex evolving in applied flow is at the root of the onset. We study the instability using a new measuring technique, the injection of a seed vortex in rotating vortex-free counterflow, and monitor the subsequent evolution of vorticity with noninvasive NMR measurement 2 .

Spin up of the superfluid component:- In uniform rotation at an angular velocity $\Omega$ the induced applied flow is the superfluid counterflow (cf) of the normal and superfluid fractions, the relative velocity $\mathbf{v}=\mathbf{v}_{\mathbf{n}}-\mathbf{v}_{\mathbf{s}}$. The viscous normal fraction (with olive oil-like viscosity) is practically always in solid-body rotation with the container, $\mathbf{v}_{\mathrm{n}}=\boldsymbol{\Omega} \times \mathbf{r}$, while the velocity $\mathbf{v}_{\mathrm{s}}$ of the superfluid fraction is produced by the combined flow field from all quantized vortex lines and from the flow caused by the boundary conditions on the container walls. Superflow is characterized by a finite critical velocity $v_{\mathrm{c}, \exp }$, at which vortex formation starts. If rotation is started at temperatures below $T_{\mathrm{c}}$, then the vortex-free Landau state is formed first, where $\mathbf{v}_{\mathrm{s}}=0$ (in the stationary laboratory frame). When the cf velocity reaches the critical value $v_{\mathrm{c}, \exp }$ (somewhere at a rough spot on the container wall), a vortex is formed, which then evolves from a micron-size loop to a rectilinear line vortex in the center of the cylinder, aligned parallel to the rotation axis. Thereby the maximum cf velocity drops below the critical

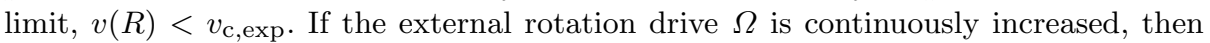
the process repeats and a central cluster of rectilinear line vortices is formed which is contained and isolated by an annular layer of vortex-free $\mathrm{cf}$ from the cylinder wall. The maximum cf velocity in the vortex-free flow is at the cylinder wall, with $v(R) \approx v_{\mathrm{c} \text {,exp }}$. Within the vortex cluster the global cf vanishes (on length scales exceeding the intervortex distance $d_{\mathrm{v}}$ ) and $\mathbf{v}_{\mathrm{S}}$ is on an average in co-rotation with $\mathbf{v}_{\mathrm{n}}$.

This is the linear well-behaved vortex formation process above $0.6 T_{\mathrm{c}}$ at high mutual friction damping, where it provides the "spin up" of the superfluid component to co-flow with the normal fraction. In macroscopic flow geometries such "quasi-intrinsic" vortex formation can be observed in superfluid ${ }^{3} \mathrm{He}-\mathrm{B}[3$, where (depending on surface roughness of the cylinder wall) the experimental $v_{\mathrm{c}, \exp }$ is perhaps only an order of magnitude smaller than the theoretical $v_{\text {c,bulk, while in }}{ }^{4} \mathrm{He}-\mathrm{II}$ this has been demonstrated only with flow through orifices of $\lesssim 1 \mu \mathrm{m}$ size. Typically in a smooth-walled cylinder $v_{\mathrm{c}, \exp } \sim 1 \mathrm{~cm} / \mathrm{s}$ at about $0.7 T_{\mathrm{c}}$ from where it decreases approximately as $v_{\mathrm{c}, \exp } \propto \sqrt{1-T / T_{\mathrm{c}}}$ towards $T_{\mathrm{c}}$. Although this is an order of magnitude smaller than the bulk liquid critical velocity $v_{\mathrm{c}, \text { bulk }} \sim 11 \mathrm{~cm} / \mathrm{s}$ (at $P=29$ bar liquid pressure), vortex-free cf can thus be maintained in metastable state up to $v(R) \lesssim v_{\mathrm{c} \text {,exp }}$, which substantially influences the NMR absorption response.

In ${ }^{3} \mathrm{He}-\mathrm{B}$ mutual friction decreases towards low temperatures almost exponentially. Thus the transition from the laminar to the turbulent flow regime at $0.6 T_{\mathrm{c}}$ is abrupt and sudden [1. Below $0.6 T_{\mathrm{c}}$ mutual friction dissipation becomes sufficiently small so that an evolving vortex may become unstable and generates a new vortex loop which then starts expanding independently. This can happen repeatedly at constant rotation 4. The experimental signature of the instability is a sudden burst of turbulence, when the density of evolving vortices grows sufficiently so that they start interacting turbulently in the bulk volume. To characterize this chain of events, we need to know how the first new loop is generated from a vortex which is evolving in applied flow. This is investigated here using the scheme outlined in Fig. 1 .

The mechanism by which the superfluid fraction is set into rotation is often called "spin up" of the superfluid component [5]. A sequence of processes is involved in spin 


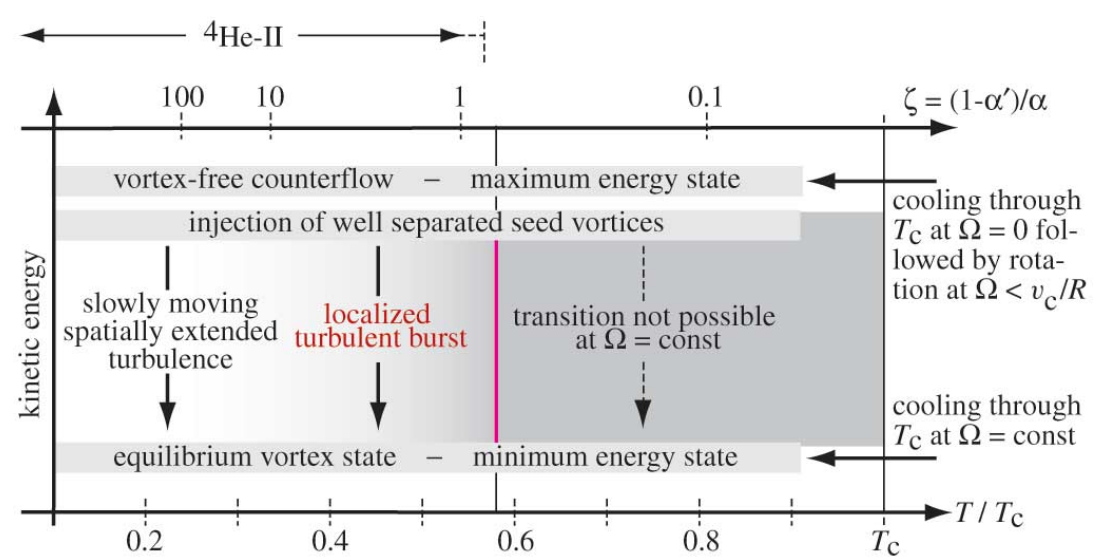

Fig. 1 (Color online) Principle of measurements on dynamic vortex generation. Well separated isolated seed vortices are introduced in rotating vortex-free cf. The initial high-energy state of vortex-free flow may then relax to the equilibrium vortex state via vortex generation processes which become possible at temperatures below the hydrodynamic transition at $0.59 T_{\mathrm{c}}$ (at 29 bar liquid pressure). The instability of a single evolving seed vortex is the first step. It is followed by a turbulent burst which is started when the density of evolving vortices is sufficient. The probability of the combined process depends on the dynamic mutual friction parameter $\zeta=\left(1-\alpha^{\prime}\right) / \alpha$ which is shown on the top together with its range of values in superfluid ${ }^{4} \mathrm{He}$.

up, one of them being the formation of new vortices. Ideally, our approach here is to study spin-up at constant rotation $\Omega$, by injecting a seed vortex in vortex-free flow. On the macroscopic scale, superfluids often mimic the behavior of viscous liquids [6]. However, as we shall see below, our superfluid spin up is rather different from the viscous flow patterns which evolve in the spin up of classical liquids [7. Owing to its high viscosity, the normal component is during the spin up in a state of laminar flow. This is an important simplification over the complicated coupled turbulent spin up of the normal and superfluid fractions which is observed in ${ }^{4} \mathrm{He}-\mathrm{II}$ at higher temperatures [6].

Generic properties of single-vortex instability:-The single-vortex instability below $0.6 T_{\mathrm{c}}$ and the phenomena which it starts in a rotating column with circular cross section have been described in Ref. 8]. Here we repeat some central features in the light of Figs. 2 and 3 before we turn to a closer characterization of the instability itself. One type of measurement on evolving vortices in a rotating cylinder is illustrated in Fig. 2. This numerical calculation with the vortex filament method 9] (see Sec. 3) monitors remanent vortices [10] in a rotating cylinder (with radius $R$ and length $L$ at constant $\Omega$ ), in a situation where the vortex instability does not occur. The purpose is to illustrate the motion of remanent vortices while they evolve from short curved vortices to rectilinear lines. The practical outcome from such a measurement is analyzed in Fig. 3 as a function of temperature.

A measurement of the kind depicted in Fig. 2 proceeds as follows: As shown in the inset, an equilibrium vortex state is first decelerated to zero rotation. After a waiting time $\Delta t$ at zero rotation, some vortices have not yet managed to annihilate. Their annihilation time is governed by mutual friction damping $\alpha(T)$ and increases rapidly with decreasing temperature. When rotation is next suddenly increased from zero to a steady value $\Omega_{\mathrm{f}}$, the remaining vortices start expanding towards their stable state as 


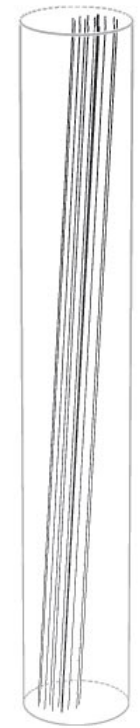

$t=0$

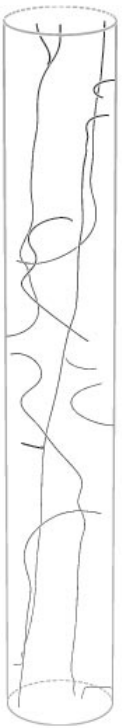

$t=600 \mathrm{~s}$
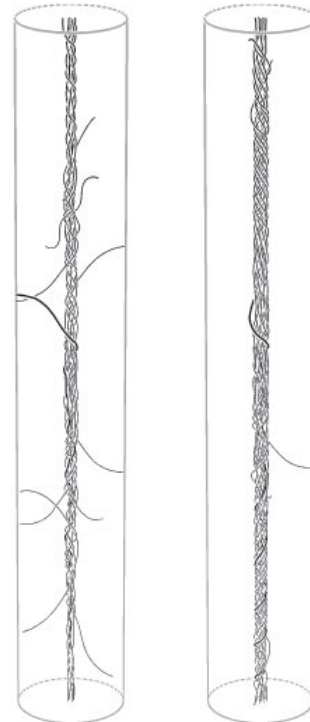

$t=800 \mathrm{~s}$

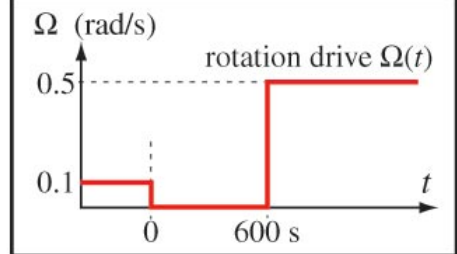

$$
\begin{aligned}
R & =3 \mathrm{~mm} \\
L & =80 \mathrm{~mm} \\
T & =0.38 T_{\mathrm{c}} \\
P & =29.3 \mathrm{bar}
\end{aligned}
$$

Fig. 2 (Color online) Numerical calculation of vortex motions in a rotating cylinder: $(t \leq 0)$ Initial state with 22 vortices at $0.1 \mathrm{rad} / \mathrm{s}$ rotation. The vortices have been artificially tilted by displacing their end points uniformly by $1 \mathrm{~mm}$ at both end plates of the cylinder, to break cylindrical symmetry. Rotation is then abruptly reduced to zero, to allow vortices to annihilate. $(t \leq 600 \mathrm{~s})$ After a waiting period $\Delta t=600 \mathrm{~s}, 12$ remanent vortices remain which are here shown at $\Omega=0$. Rotation is then increased to $\Omega_{\mathrm{f}}=0.5 \mathrm{rad} / \mathrm{s}(t \geq 600 \mathrm{~s})$ and the 12 remnants start evolving towards rectilinear lines. This requires that the vortex ends on the cylindrical wall travel in spiral motion to the respective end plates. The mutual friction parameters are $\alpha=0.18$ and $\alpha^{\prime}=0.16$ [13]. In the picture the radial lengths have been expanded by two, compared to axial distances.

rectilinear lines. This motion proceeds such that the vortex ends on the cylindrical wall travel along a spiral trajectory. During such helical motion on outer evolving vortex is wound around the straighter vortices in the center [11. The spiral motion is evident on the far right where we see a cluster of helically twisted vortices in the center of the cylinder. This state is still evolving, since ultimately also the helical twist relaxes to rectilinear lines, when the vortex ends slowly slide along the end plates of the cylindrical container [12].

Using the measuring routine in Fig. 2, we can find the onset temperature of the single vortex instability. This is done by measuring the number of rectilinear vortex lines in the final state at different temperatures. A rough value for the onset temperature can be located easily: (1) Well above $T_{\text {on }}$ the number of vortices does not change during the evolution of the remnants and thus in the final state there are only few vortices. (2) Well below $T_{\text {on }}$, in contrast, the instability occurs always and is followed by a turbulent burst which starts the evolution towards the equilibrium vortex state 8 . These two types of final states correspond to very different NMR absorption spectra. In Fig. 3 the distribution of these two final states is plotted in the immediate vicinity of $T_{\text {on }}$. Each data point corresponds to an independent measurement of the final state, after the remanent vortices have expanded in the applied cf at constant external conditions $\left(\Omega_{\mathrm{f}}\right.$, 


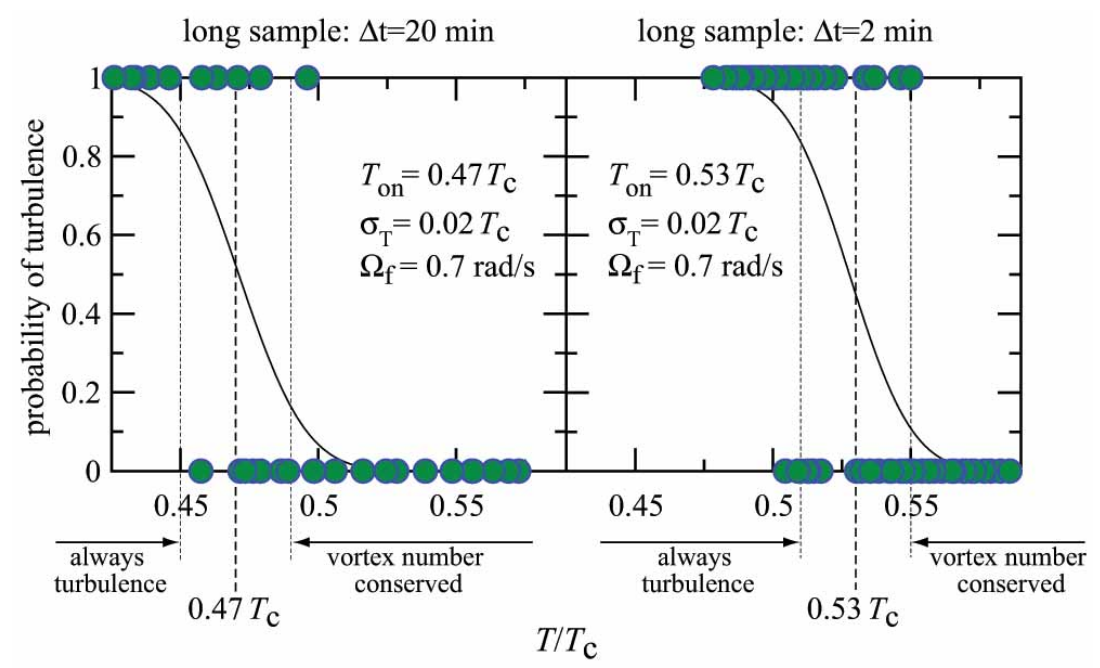

Fig. 3 (Color online) Measurements on the onset temperature $T_{\text {on }}$ of turbulence. The measurement starts from an initial state which is obtained by decelerating an equilibrium vortex state at $1.7 \mathrm{rad} / \mathrm{s}$ to $\Omega=0$ at a rate $0.01 \mathrm{rad} / \mathrm{s}^{2}$. The remaining vortices are left to annihilate 10] for a period $\Delta t$ at $\Omega=0$. Rotation is then increased to $\Omega_{\mathrm{f}}$ at a rate $0.02 \mathrm{rad} / \mathrm{s}^{2}$. When all transients have decayed the number of vortices is measured in the final steady state at $\Omega_{\mathrm{f}}$. The result is plotted as a function of temperature with $30-40$ data points per panel. The solid curve is a gaussian fit which represents the probability for turbulence with a half width $\sigma_{\mathrm{T}}=0.02 T_{\mathrm{c}}$ and centered around $T_{\mathrm{on}}$. Comparing results in the two panels for $\Delta t=20 \mathrm{~min}$ and $2 \mathrm{~min}$, we see that $T_{\text {on }}$ decreases with increasing $\Delta t$, since the number, average size, and density of remnants is reduced as $\Delta t$ increases. Parameters: $R=3 \mathrm{~mm}, L=110 \mathrm{~mm}$, and $P=29.0$ bar.

$T$, and $P$ ) and all transients have relaxed. Only in the temperature regime $T \sim T_{\text {on }}$ the final state is unpredictable. By fitting the measurements to the normal distribution we get the probability of starting the turbulent burst, the quantity plotted on the vertical scale in Fig. 3.

Two cases are compared in Fig. 3 on the right the waiting time at zero rotation is $\Delta t=2 \mathrm{~min}$, while on the left it is $\Delta t=20 \mathrm{~min}$. This turns out to yield different values for $T_{\text {on. }}$. With $\Delta t=2 \mathrm{~min}$, the number of remanent vortices $\mathcal{N}_{\mathrm{i}}$ is about ten times larger (approximately 60 at about $0.53 T_{\mathrm{c}}$ ) than with $\Delta t=20 \mathrm{~min}$ (approximately 10 at about $0.47 T_{\mathrm{c}}$ ) 10 . Because of the lower seed vortex density in the latter case, the onset moves from $0.53 T_{\mathrm{c}}$ to $0.47 T_{\mathrm{c}}$. Similarly, if $\Omega_{\mathrm{f}}$ is reduced from its value of $0.7 \mathrm{rad} / \mathrm{s}$ in Fig. 3, the onset moves to a lower temperature. This means that, to start turbulence, a sufficiently low mutual friction dissipation $\alpha(T)$ is the most important condition, but what also matters is the velocity of the applied cf and the initial number, configuration, and density of seed vortices.

The measurements in Fig. 3 are not conducted with single-vortex resolution: The number of rectilinear vortices $N$ in the final state is determined within \pm 10 lines. While the number of seed vortices is small, in the equilibrium vortex state it is $N_{\text {eq }} \approx 640$, and thus these two situations are easily distinguished. In the onset regime $T \sim T_{\text {on }}$, both failed and successful attempts for a transition to turbulence occur. In the failed attempts no increase in the number of vortices is measured, which means that only few new vortices are needed before turbulence manages to switch on. Secondly, all 
final states in Fig. 3 are either equilibrium vortex states or states with essentially no new vortices. This suggests that when turbulence is switched on, a surplus of vortices is created in the turbulent burst. Simultaneously the polarization of the vortices along the rotation axis grows to $\gtrsim 90 \%$ and the number of vortices adjusts itself approximately to that of the equilibrium vortex state.

It needs to be pointed out that the generation of new vortices in Fig. 3 is very different from regular vortex formation at temperatures above $0.6 T_{\mathrm{c}}$. The vortices in Fig. 3 are created at constant rotation $\Omega_{\mathrm{f}}$ at a low cf flow velocity $\lesssim 2 \mathrm{~mm} / \mathrm{s}$ relatively evenly along the entire column [4. If turbulence is started, then the macroscopic cf velocity ultimately drops close to zero. This means that vortex generation proceeds until completion (with the exception of a few rare examples which have been observed only in the onset regime, $T \approx T_{\text {on }}$ ). In contrast, in regular vortex formation above $0.6 T_{\mathrm{c}}$ at a rough spot on the cylinder wall (or at a wall defect called a "vortex mill" 14]), the flow becomes sub-critical after the first new vortex. A second vortex is not created (in the ideal situation [3]), unless $\Omega$ is again increased.

Thus to summarize, in contrast to the quasi-intrinsic spin-up process above $0.6 T_{\mathrm{c}}$, the single-vortex instability as the precursor to turbulence has unmistakable features, which become evident when one cools down into the intermediate temperature regime, from 0.6 to $0.3 T_{\mathrm{c}}$. Here the quasiparticle mean free path $\ell \lesssim 50 \mu \mathrm{m}$ is still smaller than the typical inter-vortex distance $d_{\mathrm{v}} \sim 0.2 \mathrm{~mm}$ and much less than the sample size $R=3 \mathrm{~mm}$. In the following we focus on the single-vortex instability and the immediately following turbulent burst.

\section{MEASUREMENTS ON SINGLE-VORTEX INSTABILITY}

Rotating flow states:- Controlled seed vortex injection and the calibration of measured NMR signals requires stability and reproducibility of different rotating flow states. Above $0.6 T_{\mathrm{c}}$ one can experimentally prepare a state with any number of rectilinear vortex lines in the central cluster up to the equilibrium number: $N \leq N_{\text {eq }}$. These are called (i) the vortex-free state $(N=0)$, (ii) a metastable vortex cluster $\left(N<N_{\text {eq }}\right)$, and (iii) the equilibrium vortex state $N \approx N_{\text {eq. }}$. When a vortex is formed as a small loop, it expands in spiral motion to a rectilinear line and becomes part of the central cluster, as seen in Fig. 2. Thereby the radius $R_{\mathrm{O}}$ of the cluster increases and the cf velocity is reduced: $v=\Omega r-\kappa N /(2 \pi r)$. Here $\kappa=h /\left(2 m_{3}\right)$ is the superfluid circulation quantum, $N$ is the number of vortices in the cluster, and $R_{\mathrm{O}}<r<R$. The cluster reaches its maximum radius in the equilibrium vortex state, where $R_{\mathrm{O}}=R-d_{\text {eq }}$ and $d_{\text {eq }} \gtrsim d_{\mathrm{v}} \approx \sqrt{\kappa /(2 \Omega)}$ is the equilibrium width of the vortex-free annulus between the cluster and the cylinder wall. Independently of the number of vortices $N$, the maximum applied flow is at the cylindrical boundary. At higher $\Omega$ (when there is no annihilation barrier [15]) the equilibrium vortex state is also the state with the maximum number of vortices (at a given value of $\Omega$ in stable conditions).

The precondition for generating these rotating states is a sufficiently high and stable critical velocity of vortex formation $v_{\mathrm{c}, \exp }$ [3. Since surface roughness reduces $v_{\mathrm{c}, \exp }$, smooth and clean container walls are important. Thus $v_{\mathrm{c}, \exp }$ is container dependent and varies even from one cool down to the next, presumably owing to frozen residual gas crystallites on the walls. Since the vortex number is reliably conserved during vortex formation processes only above $0.6 T_{\mathrm{c}}$, a particular rotating state generally has to be formed at high temperatures, but can then be cooled to low temperatures at constant 


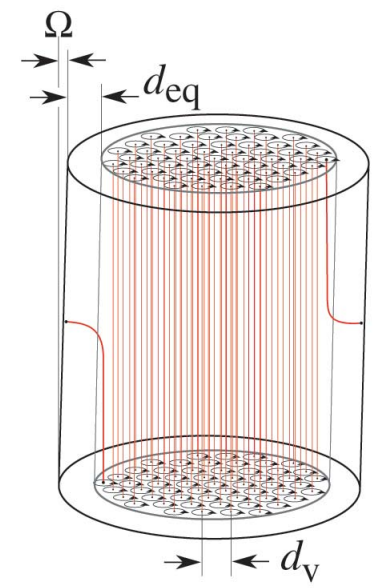

Fig. 4 (Color online) Sketch of equilibrium vortex state in a slightly tilted cylinder with two vortices connecting to the cylindrical side wall.

rotation. In particular, to have a rotating vortex-free sample at low temperatures, the procedure is to warm up first above $0.6 T_{\mathrm{c}}$, where the annihilation of remnants is rapid [10. The sample is kept there at zero rotation for 10 to $20 \mathrm{~min}$ and is then cooled down in rotation (with $\Omega<v_{\mathrm{c}, \exp } / R$ ).

The equilibrium vortex state is a particular case. Ideally, when the sample and rotation axes are perfectly aligned, all vortices in the equilibrium cluster are rectilinear. In practice, some misalignment is unavoidable, typically of order $\sim 1^{\circ}$. The result of this is that some of the outermost vortices are then curved and attached at one end to the cylindrical wall (Fig. (4). When $\Omega$ is increased, these vortices start to spiral towards rectilinear lines. Below $0.6 T_{\mathrm{c}}$ this evolution may become unstable and leads to a turbulent burst, as discussed in the context of Fig. 3 . Thus the equilibrium vortex state can be used for measurements on the single-vortex instability in a similar manner as remanent vortices.

Seed vortex injection:-By introducing seed vortices in applied flow by externally controlled means, we can monitor vortex evolution as a function of time at constant rotation. In rotating flow any vortex which is not rectilinear can be used as seed vortex, i.e. a small vortex loop or any section of a longer vortex whose configuration changes appreciably as a function of time. The active section of the seed vortex is outside the vortex cluster in the counterflow region, where the macroscopic cf velocity $v(r) \neq 0$, and is curved, with one or both ends connected to the cylindrical side wall. A number of different methods exist to start seed vortex evolution [16, 2. One example is the use of remanent vortices (Figs. 22 and 3) and a second the curved peripheral vortices of the equilibrium vortex state (Fig. 4).

An efficient injection method is based on the superfluid Kelvin-Helmholtz instability [17. of the phase boundary between the A and B phases of superfluid ${ }^{3} \mathrm{He}$. In this instability a tightly packed bundle of several approximately parallel vortex loops escapes across the AB interface into the vortex-free B-phase flow [18. This allows immediate inter-vortex interactions and starts the turbulent burst. There are several further important points to note. First, the burst follows instantaneously the KH instability and no precursory vortex generation via the single vortex instability is observed before the burst. Secondly, the burst can be localized to the immediate vicinity 
of the AB phase boundary [2]. Thirdly, the KH transition to turbulence is independent of the applied flow velocity $v(\Omega, N, R)$ [1, unlike other turbulent bursts which require the single-vortex instability as precursor 19. Most importantly however, compared to other injection methods, $\mathrm{KH}$ injection gives the highest onset temperature for turbulence. This means that the turbulent burst can happen at a higher value of vortex damping $\alpha(T)$ than where the single-vortex instability becomes possible. In KH injection the transition temperature to turbulence displays a typical narrow normal distribution [19] which is similar to that in Fig. 3 (except for the value of $T_{\text {on }}$ which is higher). This similarity suggests that plots of the transition to turbulence, like that in Fig. 3. describe the transition probability in a situation when enough vortices have already been created by the precursor mechanism so that turbulence can switch on. For these reasons $\mathrm{KH}$ injection is at present the process which is believed to identify most clearly the hydrodynamic phase transition between regular and turbulent vortex dynamics.

Finally, we note that the capture reaction of a thermal neutron in ${ }^{3} \mathrm{He}-\mathrm{B}$ releases vortex rings in a predictable manner into vortex-free $\mathrm{cf}[20$. Vortex formation is here explained to happen via the Kibble-Zurek mechanism [21], during a sudden temperature quench from the normal phase into the superfluid. This process can be reliably adjusted to emit only one single vortex ring into vortex-free B-phase flow. The telltale observation is that even this single seed vortex ring manages to start the turbulent burst at sufficiently low mutual friction dissipation [22].

For the present purpose, to investigate the single-vortex instability, we need an injection technique where the seed vortices are initially far apart at low applied cf velocity. We can use either remanent vortices [10] or the curved peripheral vortices of the equilibrium vortex state, when the sample and rotation axes are not perfectly aligned [15. In both cases rotation is increased rapidly from the initial state at $\Omega_{\mathrm{i}}$ to a final constant value $\Omega_{\mathrm{f}}$ where the evolution is recorded at constant external conditions. For remanent vortices the initial state is at zero rotation, $\Omega_{\mathrm{i}}=0$, while in the case of the equilibrium vortex state $\Omega_{\mathrm{i}}$ has some constant low value. In a strict sense these two methods do not represent injection into flow at constant cf velocity, like the KelvinHelmholtz instability or the Kibble-Zurek mechanism in neutron irradiation. However, in practice they achieve the same result, namely placing evolving vortices in rotating cf. In the onset regime, $0.3 T_{\mathrm{c}}<T \leq 0.6 T_{\mathrm{c}}$, the probability of turbulence depends primarily on the final rotation velocity $\Omega_{\mathrm{f}}$ and only weakly on the acceleration $\dot{\Omega}$ used to reach $\Omega_{\mathrm{f}}$. We use $\dot{\Omega} \sim 0.02 \mathrm{rad} / \mathrm{s}^{2}$, which in practice mimics a step increase to $\Omega_{\mathrm{f}}$.

Experimental setup:- The measurements are performed in a rotating nuclear demagnetization cryostat in which the liquid ${ }^{3} \mathrm{He}$ sample can be cooled below $0.2 T_{\mathrm{c}}$ in rotation up to $3 \mathrm{rad} / \mathrm{s}$. The temperature is determined from the frequency shifts in the NMR spectra 23, 24 and below $0.3 T_{\mathrm{c}}$ from the damping of a quartz tuning fork oscillator 25]. The sample container (Fig. 5) is a fuzed quartz tube of radius $R=3 \mathrm{~mm}$ and length $L=110 \mathrm{~mm}$, filled with liquid ${ }^{3} \mathrm{He}$ at a pressure of $P=29 \mathrm{bar}$. An aperture of $0.75 \mathrm{~mm}$ diameter in the center of the bottom end plate restricts the flow of vortices into the sample from the heat exchanger volume below.

The continuous-wave NMR absorption line shape (see insert in Fig. 6) is measured non-invasively with two detector coils at both ends of the sample cylinder. The signal is recorded with constant frequency excitation by sweeping the polarizing magnetic field. The number of vortex lines $N$ in the central vortex cluster is obtained from the NMR line shape either experimentally, by comparing to a reference spectrum which has been formed with a known number of vortices [22, or theoretically, by comparing to a cal- 


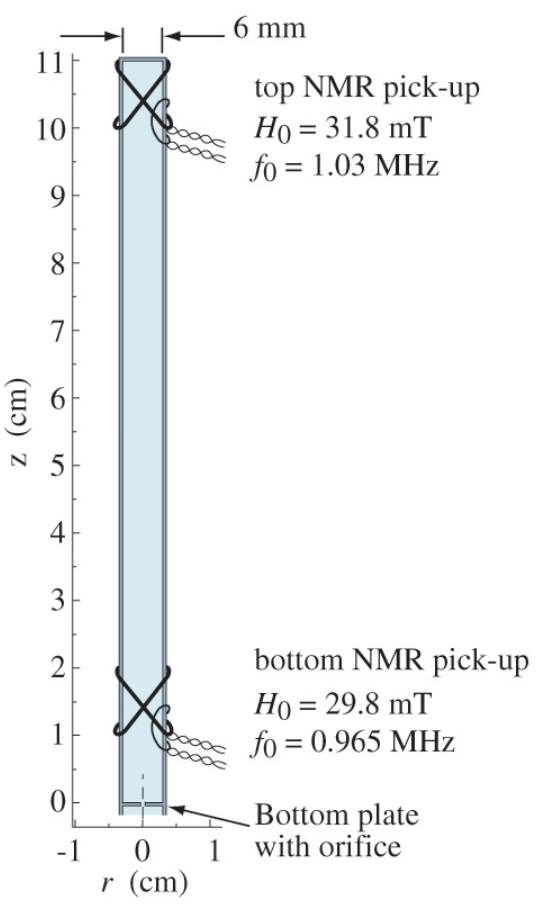

Fig. 5 (Color online) ${ }^{3} \mathrm{He}$ sample setup. The oval $2 \times 15$-turn superconducting NMR tank circuit coils are connected inductively via a two-turn pick-up loop to a room temperature preamplifier. This provides weak coupling to the pick-up coil, so that the $L C$ resonator is not excessively loaded and a Q-value of $\sim 6000$ is achieved. Two solenoidal superconducting magnets 22] (which are not shown) provide the homogeneous axially oriented polarizing fields for NMR.

culated reference, obtained from calculations of the order parameter texture 26 . The latter method is applied in this work with an overall accuracy which we estimate to be within $\pm 30 \%$. The calibrations are affected by the misalignment between the rotation and sample axes. It was measured to be $0.64^{\circ}$. In the following we make use of this experimental artifact which breaks cylindrical symmetry and makes the equilibrium vortex state at some low initial rotation velocity $\Omega_{\mathrm{i}} \neq 0$ useful as a reproducible source of seed vortices.

Such measurements are possible only if the critical velocity $v_{\mathrm{c}, \exp }(T, P)$ is stable and well-behaved. Since surface defects and dirt on the cylinder wall act as sites for nucleation, pinning, and even trapping of vortices, the quartz walls are carefully etched and cleaned. In spite of this some variation in critical velocity is observed from one cool down to the next, indicating that perhaps frozen gas particles are involved. The sample container in Fig. 5 has been in continuous use since a few years, with occasional warm ups to liquid nitrogen temperatures to clean the dilution refrigerator circulation from air plugs or to room temperature to modify the experiment. During the last 12 months $\sim 90 \%$ of cool downs at $0.8 \mathrm{rad} / \mathrm{s}$ to below $0.20 T_{\mathrm{c}}$ remain vortex-free, while at $0.9 \mathrm{rad} / \mathrm{s}$ only $\sim 20 \%$ of such attempts are successful. Before that, the same container could be regularly cooled down in the vortex-free state at $1.2 \mathrm{rad} / \mathrm{s}$. The exclusion of isolated surface defects which trap vortices is no simple task for a large cylinder like that in Fig. 5. Note that below $0.3 T_{\mathrm{c}}$ even a single expanding remnant may start a turbulent 


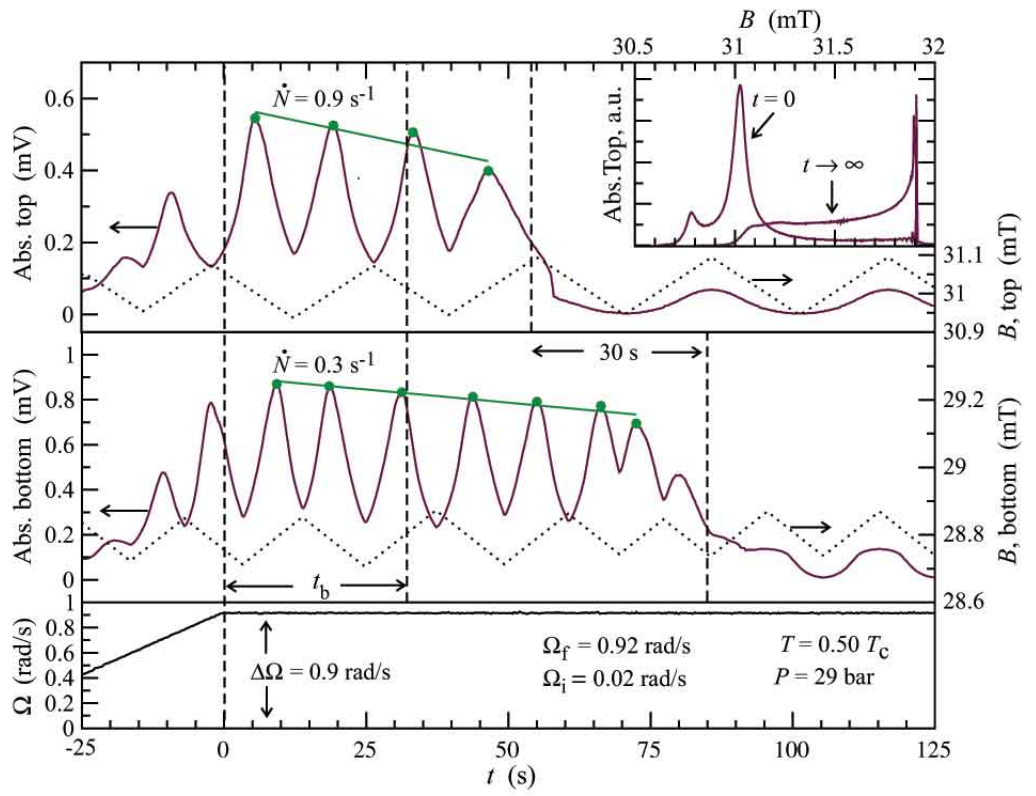

Fig. 6 (Color online) NMR record of vortex generation in applied cf. (Insert) NMR absorption line shapes at $0.51 T_{\mathrm{c}}$ and $\Omega_{\mathrm{f}}=0.92 \mathrm{rad} / \mathrm{s}$. The two line shapes represent: (i) The state after increasing rotation from the initial equilibrium vortex state at $\Omega_{\mathrm{i}}=0.02 \mathrm{rad} / \mathrm{s}$ to $\Omega_{\mathrm{f}}$. Here the total number of vortices is still close to the initial value $N_{\mathrm{i}} \approx 5$. This state is distinguished by the large shifted cf peak on the left. (ii) Final equilibrium vortex state with $N_{\text {eq }} \approx 840$ (at $t>500 \mathrm{~s}$ ), which is marked by increased absorption bordering to the Larmor edge on the right. Both line shapes have been measured with the top detector. (Main Panel) The $c f$ peak height as a function of time after increasing rotation to $\Omega_{\mathrm{f}}$ at $0.50 T_{\mathrm{c}}$ (which is below $\left.T_{\mathrm{on}}=(0.54 \pm 0.02) T_{\mathrm{c}}\right)$. The reduction in peak height represents the gradual increase in the number of vortex lines in the central cluster. The outputs from the top and bottom detectors are shown. These are not identical since the vortex formation rates $\dot{N}$ and the moments in time when the peak heights collapse may differ along the sample. The sawtooth waves represent the NMR field sweeps around the location of the cf peak which is shifted far from the Larmor value. (Bottom) Rotation drive $\Omega(t)$ as a function of time. Time $t=0$ is placed at the point where the final rotation velocity $\Omega_{\mathrm{f}}$ is reached.

burst and will then transfer the sample to the equilibrium vortex state. Probably the maximum vortex-free flow is here limited by isolated bad spots of dirt or defects on the cylindrical wall where vortices can be trapped as small loops, while they try to annihilate in zero flow. A trapped loop has a certain critical cf velocity at which it can start expanding. This velocity depends on the radius of the loop and its orientation. Thus the trapping site controls the flow velocity where the first trapped remnant starts evolving. During annihilation the isolated traps are randomly loaded with a remanent loop and thus the critical velocity varies from one measurement to the next.

Measuring procedure:- In the present work the evolving seed vortices are either remanent vortices (see Fig. 2p or vortices curving to the cylindrical side wall in the equilibrium vortex state (see Fig. 4). Accordingly, no long temperature sweeps are needed here. The rotation drive $\Omega$ is simply changed at constant temperature according to a protocol which for remanent vortices is shown in the inset of Fig. 2 The initial state is first formed by decelerating from high rotation with a large number of vortices 


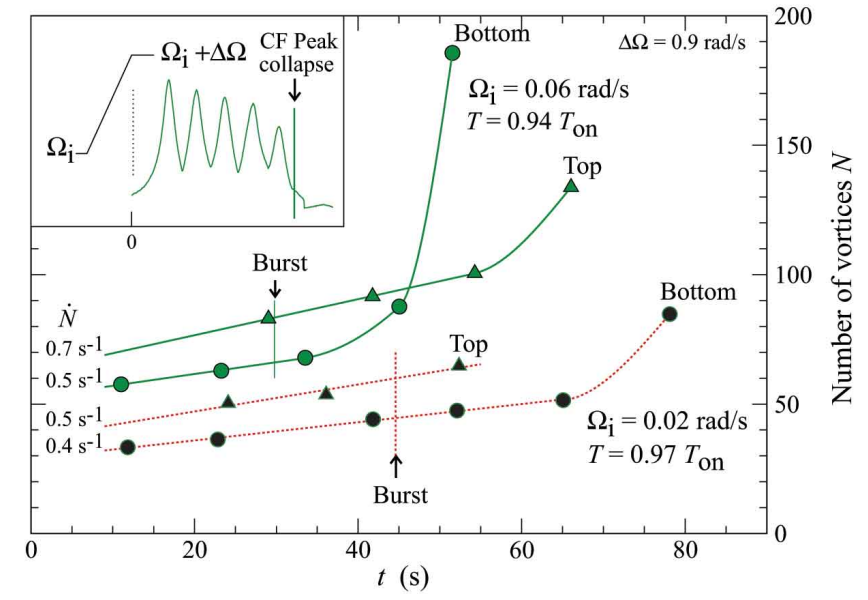

Fig. 7 (Color online) Number of vortex lines $N(t)$ in central vortex cluster as a function of time during continuous vortex formation owing to the single-vortex instability. Two measurements are shown which start from equilibrium vortex states at $\Omega_{\mathrm{i}}=0.02$ and $0.06 \mathrm{rad} / \mathrm{s}$. The calculated total number of vortices $N_{\mathrm{i}}$ is initially approximately 5 and 33, respectively, while the number of seed vortices $\mathcal{N}_{\mathrm{i}}$, which connect to the cylindrical wall, is $3-4$ in the former and about 20 in the latter case. Owing to difficulties in initial equilibration and the presence of a finite annihilation barrier [15] at $\Omega_{\mathrm{i}}=0.02 \mathrm{rad} / \mathrm{s}$, the extrapolated number of initial vortices appears to be $3-4$ times larger than the calculated estimate at very low velocities like $0.02 \mathrm{rad} / \mathrm{s}$. The vertical arrows indicate when the turbulent burst occurs in these two examples. As in Fig. 6 the burst does not take place inside either of the detector coils, but somewhere else in the long column. Since $T_{\text {on }}$ increases with $\mathcal{N}_{\mathrm{i}}, T_{\text {on }}$ has a different value for the two cases in this figure: it is $0.538 T_{\mathrm{c}}$ for $\Omega_{\mathrm{i}}=0.02 \mathrm{rad} / \mathrm{s}$, while it is $0.551 T_{\mathrm{c}}$ for $\Omega_{\mathrm{i}}=0.06 \mathrm{rad} / \mathrm{s}$. (Insert) Rotation drive $\Omega(t)$ and cf peak height measured with the bottom coil at $0.94 T_{\mathrm{on}}$. Note that Figs. 3, 6, 7 and 10 - 13 illustrate the single-vortex instability in a long column (with length/diameter $\sim 20$ ), which is sampled with two detectors, to record the time evolution simultaneously in two places. Figs. 8 ,9 and 14 in turn, describe the situation when the column is divided with an A-phase separation layer [17 in two independent samples with length/diameter $\sim 10$, so that the initial equilibration time to the equilibrium vortex state at $\Omega_{\mathrm{i}}$ becomes shorter.

to some low rotation velocity $\Omega_{\mathrm{i}}$, where rotation is maintained constant for a period $\Delta t$. If $\Omega_{\mathrm{i}}=0$, then the waiting period $\Delta t$ at zero applied flow controls the number of remanent vortices 10, as seen in Fig. 3 If $\Omega_{\mathrm{i}} \neq 0$, then we generally choose $\Delta t=300 \mathrm{~s}$, which allows the vortex array to approach closer to its equilibrium state. To start the single-vortex instability, rotation is next increased by a fixed increment $\Delta \Omega$ at $\dot{\Omega} \sim 0.02 \mathrm{rad} / \mathrm{s}^{2}$ to $\Omega_{\mathrm{f}}$, where it is kept constant and the evolution is recorded. In Fig. 6 the NMR response is shown for an example case in the onset regime $T \approx T_{\text {on }}$, where vortex generation starts spontaneously and is finally terminated in a turbulent burst.

The global superfluid cf from the rotation increase to $\Omega_{\mathrm{f}}$ produces a large absorption peak in the NMR spectrum which is shifted far from the Larmor resonance. In the main panel of Fig. 6 the height of this cf peak is monitored. The reduction in peak height as a function of time measures the increase in the number of vortices $N$ in the central cluster. Well above $T_{\text {on }}$ the cf peak height remains constant, as no new vortices are generated, but in the onset regime $T \sim T_{\text {on }}$ the height may decrease continuously, as seen in this example in Fig. 6. The initial slow rate of height reduction we attribute to vortex generation by the single-vortex instability. According to the calculated calibrations of 


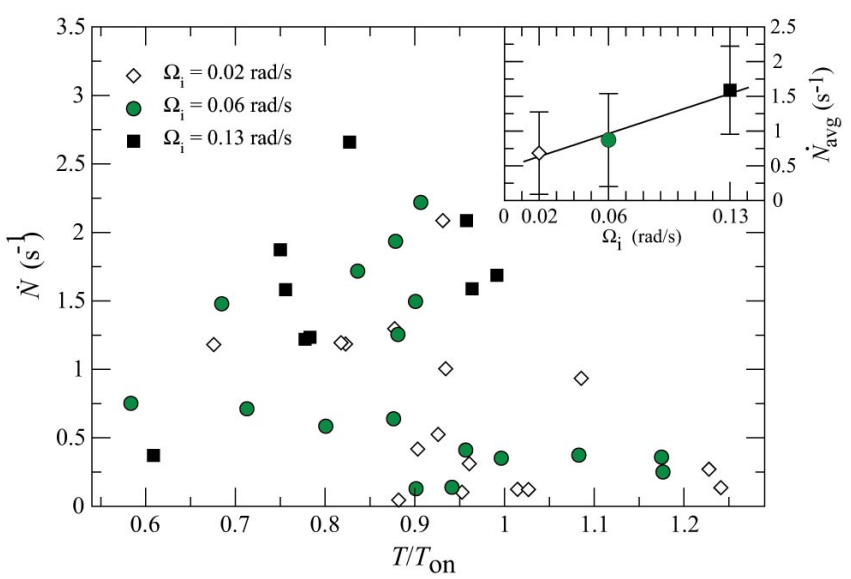

Fig. 8 (Color online) Initial rate of vortex generation, $\dot{N}($ at $t=0$ ), as a function of normalized temperature $T / T_{\text {on }}$ for events with long precursory vortex generation $\left(t_{\mathrm{b}} \gtrsim 20 \mathrm{~s}\right)$. These measurements are performed with the sample divided in independent top and bottom sections, separated by a narrow layer of ${ }^{3} \mathrm{He}-\mathrm{A}$ as barrier 2 . Initially both sections are in the equilibrium vortex state at $\Omega_{\mathrm{i}}$. To start the generation of new vortices, a rapid increase in rotation by $\Delta \Omega=0.7 \mathrm{rad} / \mathrm{s}$ is applied. The plot shows that at the final velocity $\Omega_{\mathrm{f}}=\Omega_{\mathrm{i}}+\Delta \Omega$ events with a measurable slow $\dot{N}$ are all in the onset regime $T \approx T_{\text {on }}$ : at high temperatures $T / T_{\text {on }}>1.2$ no cases of dynamic vortex generation were observed, while at low temperatures $T / T_{\text {on }}<0.6$ all measurements ended in a turbulent burst which developed too fast to provide a measurement of $\dot{N}$. (Insert) The average $\dot{N}$ of the data in the main panel plotted as a function of $\Omega_{\mathrm{i}}$. Thus $\dot{N}$ increases approximately linearly with $\Omega_{\mathrm{i}}$, in other words with the initial number of seed vortices $\mathcal{N}_{\mathrm{i}}$. The seed vortices are here the curved peripheral vortices of the equilibrium vortex state which at one end connect to the cylindrical side wall. As before, the measured value of $T_{\mathrm{on}}$ is different for each of the three values of $\Omega_{\mathrm{i}}$ in this figure. Also, vortex generation by the single-vortex instability is a stochastic event; this is the origin for the scatter.

the cf peak heights, the measured $\dot{N}$ corresponds to adding a rectilinear vortex line every few seconds to the central cluster. The final sudden collapse in height (after about $50 \mathrm{~s}$ in the top and $85 \mathrm{~s}$ in the bottom detector) marks the arrival of the equilibrium number of vortices to the respective detector coil.

The collapse of the cf peak is the signal that the turbulent burst has occurred. From the site of the burst a vortex front propagates both up and down along the rotating column 8 . When the front passes through a detector coil, the cf peak height drops to zero. Above $0.4 T_{\mathrm{c}}$ the longitudinal propagation velocity $V_{\mathrm{F}}$ of the front is approximately the same as that of the end point of a single vortex while it spirals along the cylindrical wall [27, $v_{\mathrm{L} z} \approx \alpha \Omega R$ (in an originally vortex-free rotating column). Recently measurements 28 on the front velocity were extended to temperatures below $0.2 T_{\mathrm{c}}$. Using these later values of $V_{\mathrm{F}}=\alpha_{\mathrm{eff}} \Omega R$ and correcting them for the momentary number of vortex lines $N$ in the central cluster around which the front spirals, $V_{\mathrm{F}} \approx$ $\alpha_{\text {eff }} v(\Omega, N, R)$, we calculate from the time delay between the collapse of the cf peaks in the top and bottom coils the time $t_{\mathrm{b}}$ and location $z_{\mathrm{b}}$ of the turbulent burst. In the example of Fig. 6 the measured delay of $30 \mathrm{~s}$ places the burst at a height $z_{\mathrm{b}}=76 \mathrm{~mm}$ above the orifice at time $t_{\mathrm{b}}=32 \mathrm{~s}$ (measured from the moment when the rotation drive reached $\Omega_{\mathrm{f}}$ ).

The analysis of the measured turbulent bursts allows us to conclude that multiple bursts, which would occur almost simultaneously, but in different locations along the 


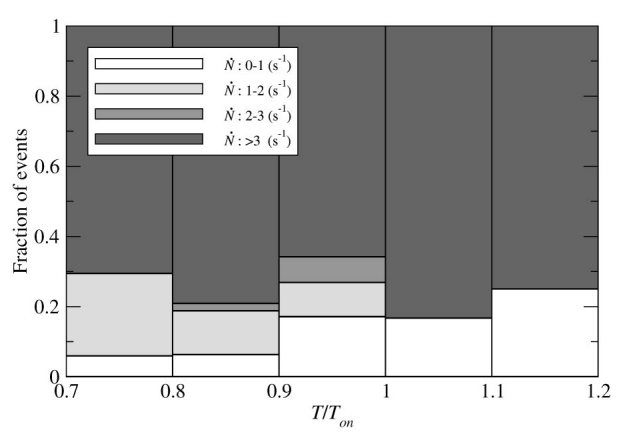

Fig. 9 (Color online) $\dot{N}$ data from the onset regime in Fig. 8 arranged independently of the $\Omega_{\mathrm{i}}$ value in four bins $\left[\left(0<\dot{N} \leq 1 \mathrm{~s}^{-1}\right),(1,2),(2,3)\right.$ and, $\left.\left(N>3 \mathrm{~s}^{-1}\right)\right]$ in different intervals of the normalized temperature $T / T_{\text {on }}$. This histogram of 140 data points characterizes the tail of the $\dot{N}$ distribution towards slow rates as a function of temperature and illustrates the frequency at which such processes occur around $T_{\text {on }}$. Although slow rates $\left(\dot{N}<3 \mathrm{~s}^{-1}\right)$ are virtually nonexistent below $0.6 T_{\mathrm{on}}$, this data set does not display a clearly increasing rate with decreasing temperature (after averaging over $\Omega_{\mathrm{i}}$ ).

rotating column, are not frequent in the onset regime and clear candidates of such events have not been identified. This conclusion is based on the continuous well-behaved behavior of the measured data on $V_{\mathrm{F}}, t_{\mathrm{b}}$, and $z_{\mathrm{b}}$. We believe that in the onset regime the probability of the turbulent burst is still small and the propagation of the vortex front so rapid that it is unlikely for bursts to start at two different locations in sufficiently close proximity in time.

The central characteristic of the single-vortex instability in Fig. 6] is the slowly decreasing cf peak height which measures the rate of vortex formation $N$. A sufficiently long period of slow peak height decay for this type of measurement is observed only in the onset regime, $T \sim T_{\text {on }}$. At lower temperatures the burst time $t_{\mathrm{b}}$ becomes very short after any small rotation increase $\Delta \Omega$ and our measurement too slow for resolving such events. The instability is easier to monitor at lower pressures where longer burst times $t_{\mathrm{b}}$ are observed 44. In Fig. 6. $T_{\mathrm{on}}=0.54 T_{\mathrm{c}}$ is defined as the average of a series of measurements on the transition to turbulence, which fit a normal distribution with a half width $\sigma_{\mathrm{T}}=0.02 T_{\mathrm{c}}$. Here and in all later examples, $T_{\mathrm{on}}$ is measured in each case separately for the appropriate conditions of that particular measuring situation, as was done in Fig. 3.

To summarize, the collapse of the cf peak height in Fig. 6 is caused by the arrival of the vortex front. The front moves with a velocity which depends on the number of vortices $N$ in the central cluster at height $z$, before the front is about to pass at $z$. In an ideal case, where the vortex instability occurs continuously and randomly in the sample, one might expect that the site of the burst is randomly distributed along the $z$ axis. Varying $\Omega_{\mathrm{i}}$, we can change in a controlled manner the number of curved seed vortices $\mathcal{N}_{\mathrm{i}}$ which connect initially to the cylindrical side wall. In addition, by changing $\Delta \Omega$ or temperature, we control respectively the applied flow velocity $v(\Omega, R, N)$ or the damping $\alpha(T)$. By studying the dependence of $T_{\text {on }}$ on these variables one can analyze how they influence the onset of turbulence (compare Figs. 3 and 14). Next we are going to focus on the properties of the precursor, represented by the approximately linear $\dot{N}(t)$ in Fig. 6. by examining its characteristics in the onset regime $T \approx T_{\text {on }}$, such as the vortex formation rate $\dot{N}$, and the distributions of the burst time $t_{\mathrm{b}}$ and the burst locations $z_{\mathrm{b}}$. 


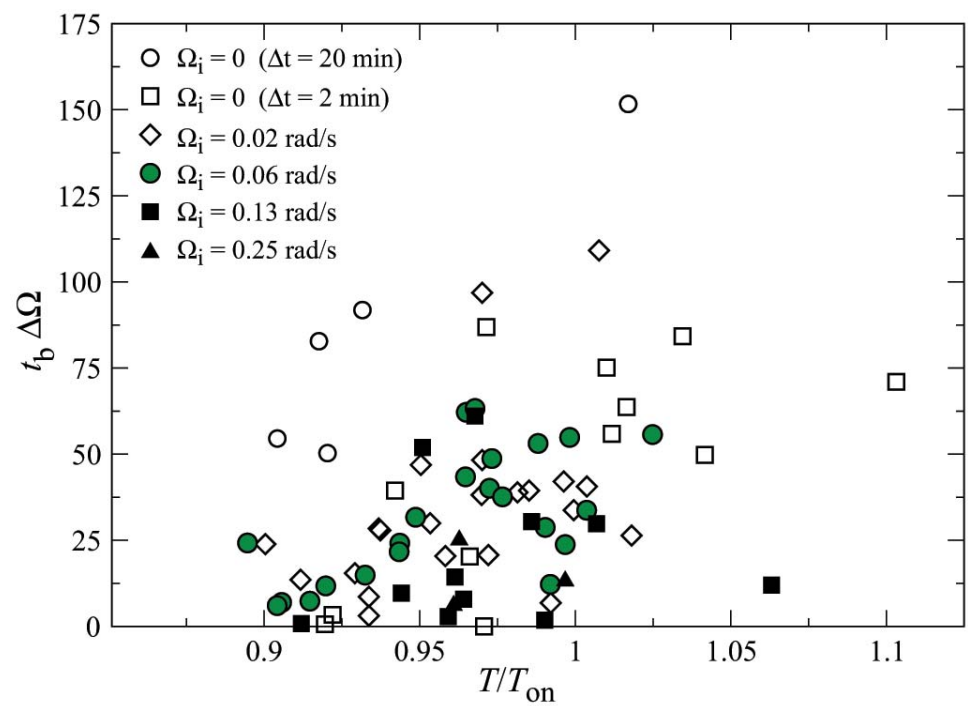

Fig. 10 (Color online) Time $t_{\mathrm{b}}$ needed to start the turbulent burst during continuous vortex generation at constant rotation $\Omega_{\mathrm{f}}=\Omega_{\mathrm{i}}+\Delta \Omega$, plotted as a function of temperature around the onset $T \approx T_{\mathrm{on}}$. The data have been collected at five different values of $\Omega_{\mathrm{i}}$ and three values of $\Delta \Omega=0.7,0.9$, and $1.3 \mathrm{rad} / \mathrm{s}$. Since $T_{\text {on }}$ depends on both $\Omega_{\mathrm{i}}$ and $\Delta \Omega$, in each case the appropriate measured value of $T_{\mathrm{on}}$ is used for normalizing the temperature axis. The data for $\Omega_{\mathrm{i}}=0$ come from measurements on remanent vortices with $\Delta \Omega=0.7 \mathrm{rad} / \mathrm{s}$, as in Fig. 3. No A-phase barrier field is applied in these measurements and thus the sample is here twice as long as in Fig. 8 . The plot suggests that on average $t_{\mathrm{b}}$ increases with normalized temperature $T / T_{\text {on }}$ and decreases with $\Omega_{\mathrm{i}}$.

Experimental results:-Fig. 7 shows two examples, after conversion from cf peak height to vortex number $N(t)$. When vortex generation proceeds slowly, the rate $\dot{N}$ is initially of order 1 vortex/s, but increases at later times and becomes more nonlinear, until the vortex front passes through the coil and $N$ jumps to nearly $N_{\text {eq }}$. As seen in Fig. 7. generally the initial rate $\dot{N}(t=0)$ increases with increasing $\Omega_{\mathrm{i}}$, since the number of seed vortices $\mathcal{N}_{\mathrm{i}}$, which connect to the cylindrical side wall in the initial equilibrium vortex state, increases with $\Omega_{\mathrm{i}}$.
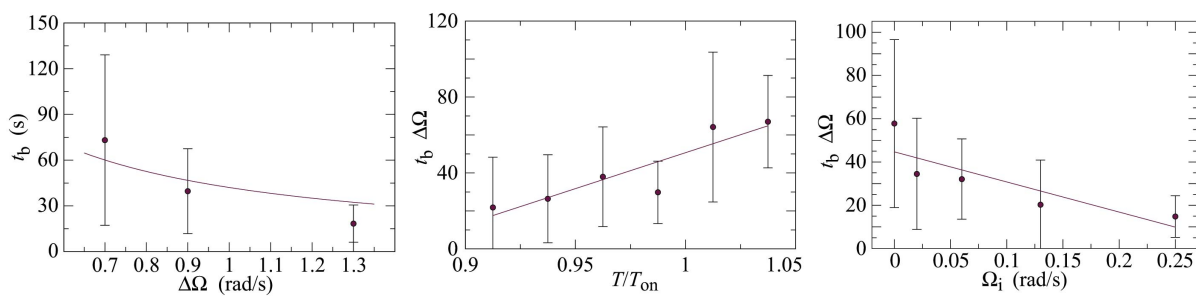

Fig. 11 (Color online) Burst time $t_{\mathrm{b}}$, averaged for the data in Fig. 10 and analyzed for a dependence on the applied flow velocity $\sim \Delta \Omega$ (left), on the normalized temperature $T / T_{\text {on }}$ (center), and on the number of seed vortices $\sim \Omega_{\mathrm{i}}$ (right). The averaging has been done by dividing the data points in bins as a function of the respective variable and by denoting the standard deviation in each bin with uncertainty limits. For instance, on the left we see that $t_{\mathrm{b}}$ decreases with increasing flow velocity $v(\Omega, N, R)$, since this velocity is roughly expressed by the rotation increase $\Delta \Omega$. Here the solid curve is the average $\left\langle t_{\mathrm{b}}\right\rangle=42 / \Delta \Omega$. 


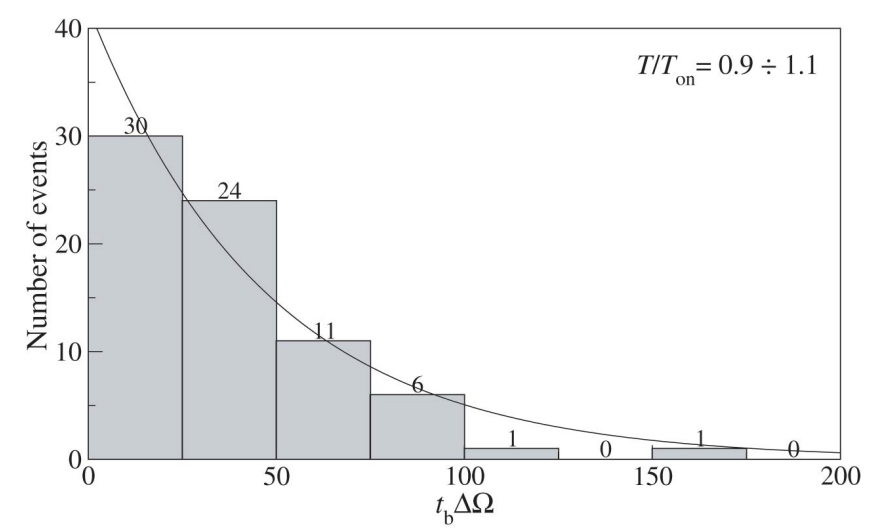

Fig. 12 (Color online) Temporal distribution of turbulent bursts in the onset temperature regime. The burst time data from Fig. 10 is here organized as a histogram for events where $t_{\mathrm{b}}>20 \mathrm{~s}$ is long enough to be resolved. The solid curve is an approximation to the tail of the probability distribution for $t_{\mathrm{b}}$ and represents the fitted exponential $\propto \exp \left(-t_{\mathrm{b}} \Delta \Omega / 47\right) \approx$ $\exp \left(-t_{\mathrm{b}} /\left\langle t_{\mathrm{b}}\right\rangle\right.$, where $\left\langle t_{\mathrm{b}}\right\rangle$ is the average $t_{\mathrm{b}}$ at given $\Delta \Omega$ (Fig. 11. left).

More statistics on the precursor are presented in Fig. 8 , collected from measurements similar to those in Fig. 6. The initial rate of vortex generation $\dot{N}(t=0)$ is compared here in the onset regime $T \sim T_{\mathrm{on}}$ for different $\Omega_{\mathrm{i}}$ and thus for different number of evolving vortices $\mathcal{N}_{\mathrm{i}}$, keeping the rotation drive $\sim \Omega_{\mathrm{f}}-\Omega_{\mathrm{i}}=\Delta \Omega=0.7 \mathrm{rad} / \mathrm{s}$ constant. In the inset the average of the measurements is seen to depend roughly linearly on $\Omega_{\mathrm{i}}$, as was concluded in the context of Fig. 7 The data in Fig. 8 includes only events with long burst times $t_{\mathrm{b}} \gtrsim 20 \mathrm{~s}$, where the slope of the cf peak height with time can be clearly determined (while events with $t_{\mathrm{b}}<20 \mathrm{~s}$ can be counted, but the value of $t_{\mathrm{b}}$ is not resolved). The data is shown again by the histogram of Fig. 9 but now including also the fast events, where $t_{\mathrm{b}}<20$ s and $\dot{N}>3$ vortices/s. Fig. 9 shows that, on average, $\dot{N}$ increases in this data set with decreasing temperature. In addition we observe no events with a slow measurable $\dot{N}$ below $0.6 \leq T / T_{\text {on }}$. We thus have to conclude that extended precursory vortex generation with a burst time $t_{\mathrm{b}} \gtrsim 20 \mathrm{~s}$ can only be observed in the onset temperature regime $T \sim T_{\text {on }}$.

In Fig. 10 a different data set with measurements on the burst time $t_{\mathrm{b}}$ is examined. Since our measurement captures efficiently only events with long burst times, Fig. 10 represents the tail $\left(t_{\mathrm{b}} \geq 20 \mathrm{~s}\right)$ of the burst-time distribution. These 73 data points are almost half of all the measured turbulent events in the temperature interval $0.9<$ $T / T_{\text {on }}<1.1$ in the present data set. Thus events with $t_{\mathrm{b}} \gtrsim 20 \mathrm{~s}$ and prominent slow vortex formation are relatively frequent close to $T_{\mathrm{on}}$. The equations of vortex motion roughly scale with the effective rotation drive, which in this case is $\sim \Delta \Omega$. As the data have been collected with three different values of $\Delta \Omega$, we plot the result as $t_{\mathrm{b}} \Delta \Omega$ which corrects for differences in the drive sufficiently well. The main conclusions to be drawn from this data are illustrated in the three plots of Fig. 11] where the averages are shown as a function of the rotation increase $\Delta \Omega$, normalized temperature $T / T_{\text {on }}$, and the rotation velocity of the initial equilibrium vortex state $\Omega_{\mathrm{i}}$. The interpretation of these plots is that the burst time $t_{\mathrm{b}}$ decreases rapidly with increasing applied flow velocity $\sim \Delta \Omega$, increases with temperature $T / T_{\text {on }}$, and decreases with with $\Omega_{\mathrm{i}}$, i.e. the number of seed vortices $\mathcal{N}_{\mathrm{i}}$. In Fig. 12 the tail of the burst time distribution is 


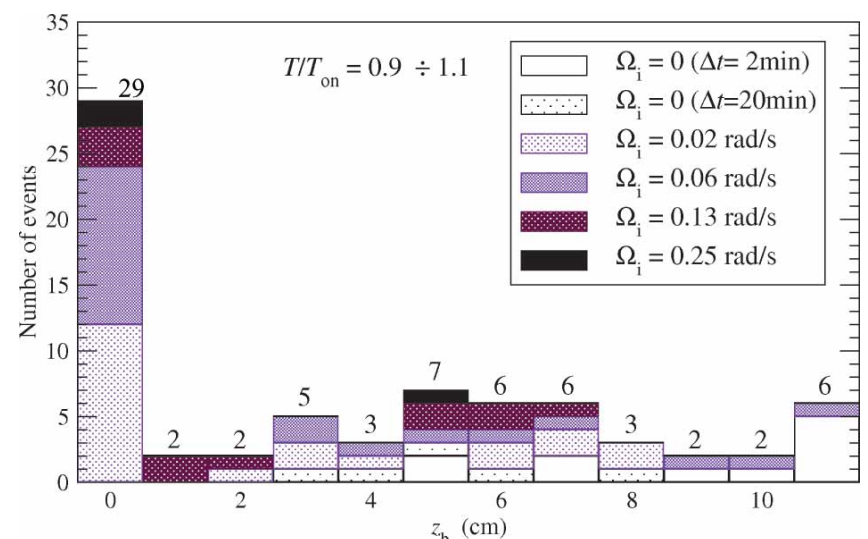

Fig. 13 (Color online) Spatial distribution $z_{\mathrm{b}}$ of turbulent bursts for the data in Fig. 10 organized as a histogram along the $z$ axis of the long sample in Fig. 5 The bursts appear to be randomly distributed, except for a strong preference for the region below the bottom coil. This additional mechanism, which enhances the probability of the turbulent burst, we assume to be associated with the presence of the orifice below the bottom coil.

shown as a histogram, indiscriminately for all $t_{\mathrm{b}}$ data in Fig. 10 As seen here, in the onset regime, $T \approx T_{\mathrm{on}}$, the probability for large burst times decreases approximately exponentially.

To summarize we conclude that both data sets, displayed in Figs. 8 and 10 illustrate that well-resolved single-vortex instability events are distinguished by slow vortex formation $\dot{N}(t=0)$ and a long burst time $t_{\mathrm{b}}$. Such events can be found (i) in the onset temperature regime, $T \approx T_{\mathrm{on}}$, (ii) by starting from a state with a small number of seed vortices $\mathcal{N}_{\mathrm{i}}$, and (iii) by keeping the applied flow velocity $(\sim \Delta \Omega)$ as small as possible.

In Fig. 13 the spatial distribution of the turbulent bursts from Fig. 10 is displayed along the $z$ axis of the sample. As expected, the location $z_{\mathrm{b}}$ of the burst is approximately evenly distributed along the column. This supports the notion that the generation of new vortices occurs randomly with equal probability along the entire cylinder. The exception is a clear preference for the region below the bottom coil. The breakdown of these events with $z_{\mathrm{b}}<10 \mathrm{~mm}$ according to their $\Omega_{\mathrm{i}}$ values shows that the orifice is a large perturbation for small vortex clusters. If $\Omega_{\mathrm{i}}=0.02 \mathrm{rad} / \mathrm{s}$, then the cluster radius $R_{\mathrm{O}} \approx R\left(\Omega_{\mathrm{i}} / \Omega_{\mathrm{f}}\right)^{\frac{1}{2}}$ is approximately equal to the radius of the orifice. Even with $\Omega_{\mathrm{i}}=0.06 \mathrm{rad} / \mathrm{s}$ the two might be comparable, since this comparison is affected by the centering of the orifice on the bottom plate and the inclination of the cylinder and rotation axes. At higher $\Omega_{\mathrm{i}}$ values the cluster apparently covers the orifice more efficiently and its perturbing effect fades away. Surprisingly, no cases of turbulent bursts are present in Fig. 13 which would have been started by remanent vortices at the orifice (i.e. with $\Omega_{\mathrm{i}}=0$ ), but this observation might change with a larger sample of measured events.

Discussion:-We thus find that the presence of the orifice promotes the probability of the single vortex instability and concentrates turbulent bursts in the section below the bottom detector coil. This is also seen in direct measurements on the individual transition temperatures $T_{\text {on }}$ of the top and bottom sections of the long sample cylinder. In Fig. 14 the cylinder has been divided in two disconnected halves with a narrow transverse layer of ${ }^{3} \mathrm{He}-\mathrm{A}$, as first discussed in Ref. [17. The AB interface acts as a barrier 2] for vortices and thus the two sections can be sampled separately with 


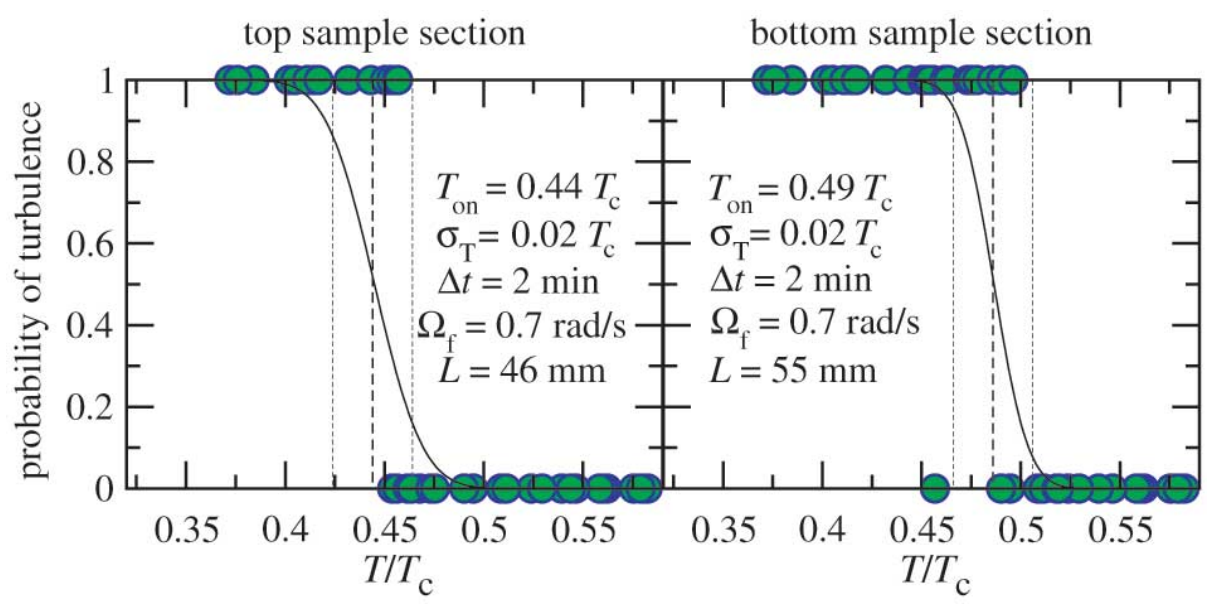

Fig. 14 (Color online) Onset temperature of turbulence for the upper and lower sections of the sample in Fig. 5 The division into these two sections is described in Ref. 10]. The measurements are performed similar to those in Fig. 3 starting from an initial state at zero rotation with remanent vortices left over after an annihilation period $\Delta t=2 \mathrm{~min}$. Comparing the two sections, we recognize that $T_{\mathrm{on}}$ is higher in the bottom section, i.e. the probability of turbulence at a given temperature is enhanced in the bottom section. The main difference between the two sections is the orifice in the lower part. This suggests that the vicinity of the orifice is particularly propitious for starting the single-vortex instability. The solid curves are normal distributions with a half width $\sigma_{\mathrm{T}}=0.02 T_{\mathrm{c}}$, centered around $T_{\mathrm{on}}$.

the detector coils at each end of the cylinder. Comparing the four $T_{\text {on }}$ distributions for remanent vortices in Figs. 3 and 14, it is seen that the $T_{\text {on }}$ values are all distinctly different, but in increasing order form a sequence which can now be explained based on the previous conclusions. These four $T_{\text {on }}$ distributions have been measured in the same set of measurements, without ever warming above dilution refrigerator temperatures. This means that they are closely comparable and representative of the same experimental environment. Nevertheless, comparing the two distributions in Fig. 14 it is evident that a relatively large difference of $0.05 T_{\mathrm{c}}$ separates the $T_{\mathrm{on}}$ values of the top and bottom sections. This difference is large enough so that the two distributions do not overlap. We conclude that the probability of the turbulent burst increases at a given temperature when the bottom section is included in the sample - or that the vicinity of the orifice is a particularly efficient environment for starting the turbulent burst.

In earlier work [29] it was proposed that a large number of vortices might leak simultaneously through the orifice as a sudden burst into the sample volume and that this is the origin for the abrupt transition from the vortex-free to the equilibrium vortex state. Below the orifice vortices are typically present in the equilibrium vortex state in most situations, since two quartz tuning fork oscillators are located there 25. These provide ample opportunity for vortex pinning and trapping. Moreover the bottom surface in this volume is a rough sintered heat exchanger. However, considering Figs. 3 and 14 together, a massive sudden leakage of vortices through the orifice appears now questionable. Comparing the bottom section in Fig. 14 to the corresponding plot for the long sample in Fig. 3 (with $\Delta t=2 \mathrm{~min}$ on the right), we see that $T_{\text {on }}$ is not determined by only the orifice, but also depends on the length of the cylinder above the orifice: The twice larger sample length $L$ of the long sample causes the onset $T_{\text {on }}$ 
to increase by $0.04 T_{\mathrm{c}}$. This feature is consistent with the general notion that in the long sample there are twice as many seed vortices to begin with, the evolving vortices spend more time spiralling along the cylinder wall, and are more likely to suffer the instability, than in the short sample. In contrast, an event with lots of vortices leaking through the orifice should be insensitive to the length of the sample above the orifice and seems less likely to explain the measurements on $T_{\text {on }}$.

Nevertheless, Fig. 13 shows that a large fraction of the turbulent bursts occur in the vicinity of the orifice and all measurements on the bottom section show a higher value of $T_{\text {on }}$ by about $0.04 T_{\mathrm{c}}$ than an equivalent measurement on the top section. This difference is visible also in Fig. 14 Thus we have to conclude that the single-vortex instability is more likely at a given temperature when the orifice is included in the sample and that the bottom section therefore has a higher onset temperature than the top. It is not clear at this point how the presence of the orifice enhances the probability of the single-vortex instability, since it affects both the initial configuration of seed vortices and their later dynamics after the rotation increase. Here both geometry as well as surface roughness could be important.

In contrast the lower value of $T_{\mathrm{on}}$ for the top section indicates that an isolated cylinder (which in this case is closed off by the AB interface barrier) displays a reduced probability for the single-vortex instability to occur, in other words the top section, with no obvious defects, is closer to an ideal cylindrical sample. A further measurement on only the top section, with a reduced number of initial seed vortices, obtained by increasing the annihilation period $\Delta t$ from $2 \mathrm{~min}$ to $20 \mathrm{~min}$, reduces $T_{\mathrm{on}}$ from $0.44 T_{\mathrm{c}}$ (Fig. 14 left) to $0.39 T_{\mathrm{c}}$. This result is what we expect, based on the examples presented above: $T_{\text {on }}$ decreases if the number of seed vortices is reduced.

To summarize, in the onset temperature regime $\left(T \approx T_{\text {on }}\right)$ the single-vortex instability progresses sufficiently slowly in about one third of the measured events so that it can be recorded with our measurement techniques. It functions as the precursor mechanism which generates new dynamically evolving vortices, until a localized turbulent burst between interacting vortices becomes possible in a short section (of length $\sim R$ ) of the rotating column. The instability depends foremost on temperature via the dynamic parameter $\zeta=\left(1-\alpha^{\prime}\right) / \alpha$, which for superfluids is the equivalent of the Reynolds number of viscous hydrodynamics, namely the ratio of the inertial and dissipative forces 2]. Our measurements in the onset regime can be interpreted in terms of the probability of a single dynamically evolving vortex to undergo the instability and to create a new vortex loop which in turn starts to evolve. At a given temperature the probability to achieve bulk turbulence depends on the applied cf velocity at the container boundary, on the length of the trajectory over which the vortex end travels along the boundary, and on the total number of vortices which simultaneously are dynamically evolving.

An earlier explanation of the onset of superfluid turbulence was provided by Klaus Schwarz who in 1993 concluded (based on his own work and that of others) that a set of several vortex mills is required to start and maintain turbulence in channel flow 30. These vortex mills need to act in parallel and have to be located close to the entrance of the flow channel. Our results now show that vortex mills are not necessary to start turbulence and that there exists a more fundamental instability mechanism, namely the single-vortex instability. In principle, the characterization of this instability in Figs. 3. and 6- 14 can be compared to simulation calculations, to reconstruct a more detailed understanding. A step towards this goal is taken in the next section, where the instability mechanism is studied in numerical calculations. 


\section{NUMERICAL CALCULATIONS ON SINGLE-VORTEX INSTABILITY}

Numerical method: Our calculations 9] are carried out using the vortex filament model introduced by Schwarz 31. With Biot-Savart integration along all vortex lines the superfluid velocity field from vortices is obtained from

$$
\mathbf{v}_{\mathrm{s}, \omega}(\mathbf{r}, t)=\frac{\kappa}{4 \pi} \int \frac{(\mathbf{s}-\mathbf{r}) \times d \mathbf{s}}{|\mathbf{s}-\mathbf{r}|^{3}} .
$$

The line integral is taken along the vortices and $\mathbf{s}(\xi, t)$ denotes the location of the vortex core at time $t$, while $\xi$ is measured along the arc length of the vortex core. In the presence of solid boundaries the total superfluid velocity field, $\mathbf{v}_{\mathrm{s}}=\mathbf{v}_{\mathrm{s}, \omega}+\mathbf{v}_{\mathrm{b}}$, is modified by the boundary induced velocity $\mathbf{v}_{\mathrm{b}}$. At a plane boundary one can use image vortices to satisfy the requirement of zero flow through the boundary, $\hat{\boldsymbol{n}} \cdot \mathbf{v}_{\mathrm{S}}=0$, where $\hat{\boldsymbol{n}}$ is the unit vector along the surface normal. More generally we obtain $\mathbf{v}_{\mathrm{b}}=\nabla \Phi$ by solving the Laplace equation $\nabla^{2} \Phi=0$ combined with the requirement that at the boundary $\hat{\boldsymbol{n}} \cdot \nabla \Phi=-\hat{\boldsymbol{n}} \cdot \mathbf{v}_{\mathrm{s}, \omega}$. No surface pinning or surface friction is included, the boundaries are assumed ideal which, as far as known, is not in contradiction with measurements. Mutual friction in the bulk superfluid is included using the equation of motion for the vortex element at $\mathbf{s}(\xi, t)$

$$
\mathbf{v}_{\mathrm{L}}=\frac{d \mathbf{s}}{d t}=\mathbf{v}_{\mathrm{s}}+\alpha \mathbf{s}^{\prime} \times\left(\mathbf{v}_{\mathrm{n}}-\mathbf{v}_{\mathrm{s}}\right)-\alpha^{\prime} \mathbf{s}^{\prime} \times\left[\mathbf{s}^{\prime} \times\left(\mathbf{v}_{\mathrm{n}}-\mathbf{v}_{\mathrm{s}}\right)\right] .
$$

where the vector $\mathbf{s}^{\prime}=d \mathbf{s} / d \xi$ is the local tangent to the vortex at the point $\mathbf{s}(\xi, t)$. For the mutual friction parameters $\alpha(T, P)$ and $\alpha^{\prime}(T, P)$ we use the 29 bar data measured in Ref. 13.

In practice, the implementation of the Biot-Savart integration is performed adaptively, i.e. the number of discretization points along a vortex in evaluating the BiotSavart integral is increased recurrently, until the required accuracy is obtained. The vortex is split along its core into line segments whose length is adjusted such that shorter segments are used in places where the vortex is more curved or the counterflow is large (enabling smaller wavelength Kelvin-waves). The smallest segment length $\Delta \xi$ limits the time step $\Delta t$ which is used to solve the time development of the tangle with the classical 4 th order Runge-Kutta method. The solution of the Laplace equation is obtained by discretizing the potential $\Phi$ within the cylinder (typical grid size, eg. radially, $\Delta r=R / 15$ ). The resulting sparse matrix equation is then solved at each time step, while the spatial derivatives are approximated with finite differences. This means that the continuity equation for the superfluid velocity is not accurately satisfied. Nevertheless, this scheme is an improvement over much of the earlier work.

To solve for $\mathbf{v}_{\mathrm{b}}$, one needs to make sure that vortices meet the boundaries perpendicularly and that in Eq. (1) one integrates along vortices which form closed loops, as noted by Schwarz [32. The latter requirement is implemented by extending the vortices, which terminate perpendicularly on the boundary, to infinity with straight vortex line sections. A vortex reconnection is performed when two vortices approach each other closer than the maximum resolution $(=\Delta \xi \sim R / 50$ typically, measured along the vortex core), provided that the resulting configuration has reduced length and represents thus a lower energy state. Generally the maximum resolution has minor effect on the results in the intermediate temperature regime. Increased resolution slows down the calculations and results in larger numbers of tiny vortex loops which in any 


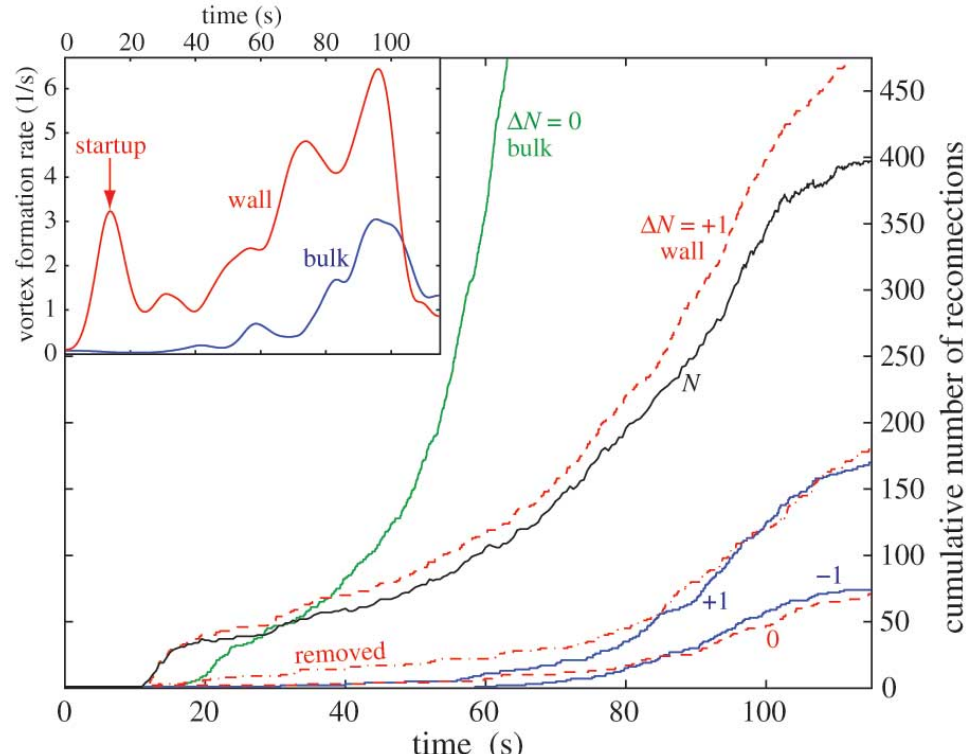

Fig. 15 (Color online) (Main panel) Calculated build up in cumulative number of vortices and their reconnections in a rotating cylinder: $(0$, solid curve) reconnections in the bulk which do not change $N,(+1$, dashed) surface reconnections which add one new vortex loop, $(\mathrm{N})$ total number of vortices, (removed, dash-dotted) small loops which form in reconnections mainly close to the cylindrical wall, but which are contracting and are therefore removed, $(+1$, solid $)$ bulk reconnections which add one vortex, $(-1$, solid) bulk reconnections which remove a vortex, $(0$, dashed) reconnections at the boundary which do not change $N$. (Insert) Averaged number of reconnections per second on the cylindrical boundary and in the bulk which add one vortex. The large initial peak in the boundary rate represents the starting burst which is required to start vortex formation. Parameters: $R=3 \mathrm{~mm}, L=10 \mathrm{~mm}, \Omega=0.9 \mathrm{rad} / \mathrm{s}$, and $T=0.35 T_{\mathrm{c}}$ (where $\alpha=0.095$ and $\alpha^{\prime}=0.082[13$ ).

case rapidly disappear owing to the finite mutual friction damping. Nevertheless, a sufficiently fine resolution is needed to display Kelvin-wave excitations, and towards low temperatures the resolution needs to increase rapidly. In solving the Laplace equation for the boundary condition, a coarser resolution must be tolerated, to avoid too large memory consumption.

Fig. 2 is an example of how calculations can be used to illustrate and interpret measurements. This calculation is performed at $0.38 T_{\mathrm{c}}$ and conserves the number of vortices during their evolution, after increasing the rotation velocity from zero to $\Omega_{\mathrm{f}}$. Comparing to Figs. 3 and 14 we note that in most experimental cases $0.4 T_{\mathrm{c}}$ is below $T_{\text {on }}$, so that the single-vortex instability would interfere and would lead to turbulence. The origin of this difference between calculations and experiments has not been resolved. To start vortex generation in the calculation, often a specially designed unstable starting configuration is required, which creates a larger number of interacting vortices [4].

Vortex generation in rotating flow:-In Fig. 15 the generation of vortices is studied in a short circular cylinder with diameter comparable to height. Account is kept of all reconnection processes as a function of time while the sample is evolving towards its final state. Vortex formation is initially started from a single vortex ring which is placed in the plane perpendicular to the rotation axis at height $0.2 \mathrm{~L}$ slightly off center, 


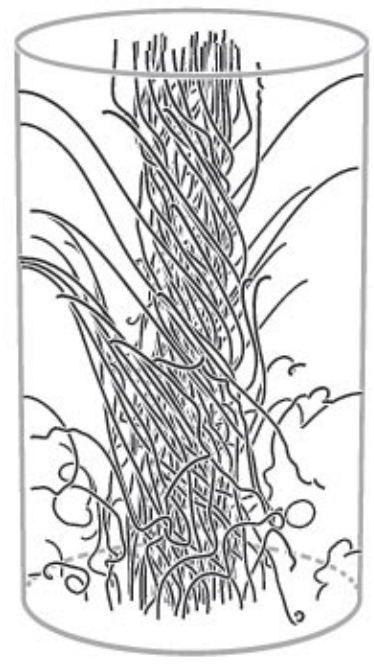

$t=50.5 \mathrm{~s}$

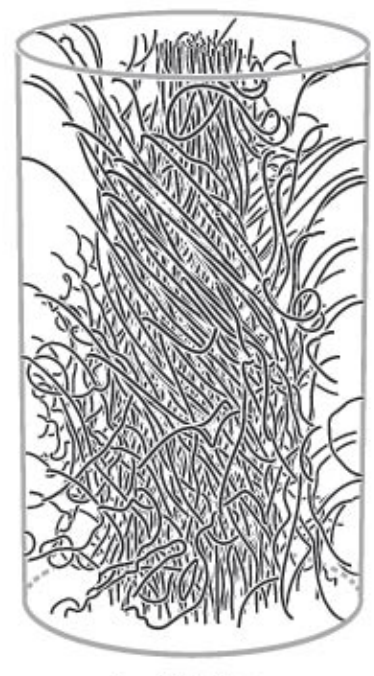

$t=77.5 \mathrm{~s}$

Fig. 16 Two snapshots from the calculation in Fig. 15 In both cases helically twisted younger vortices can be seen on the outer circumference of the cluster and older straighter vortices in the center. Primarily surface reconnections contribute to the formation of new vortices at $t<100 \mathrm{~s}$ which explains the many short loops outside both clusters.

to break cylindrical symmetry (see Ref. 4]). This is an unstable configuration where Kelvin waves of large amplitude immediately form and reconnect at the cylindrical wall. The end result is a sudden formation of roughly 30 vortices which have one end on the bottom end plate and the other moving in spiral trajectory along the cylindrical wall. After this initial burst the spontaneous evolution is followed in the calculation, the formation of new vortices is noted, and the reconnections of different type are classified.

In Fig. 16 we see snapshots of vortex configurations after $50 \mathrm{~s}$ and $80 \mathrm{~s}$. Recently formed vortices are here on the outer circumference in helical configuration, while further inside the cluster the vortices are gradually relaxing towards rectilinear lines. Outside the cluster closer to the cylindrical wall one can see loops of Kelvin waves, small separated loops with both ends of the vortex on the cylindrical wall, and even closed vortex rings (lower right corner at $t=50 \mathrm{~s}$ ).

Returning to Fig. 15, we note that after the initial burst of the first $\sim 30$ vortices $N$ increases first gradually, but after about $50 \mathrm{~s}$ the rate $\dot{N}$ picks up. During the first $50 \mathrm{~s}$ reconnections in the bulk do not contribute to the generation of new vortices, but later such processes also start appearing. Surprisingly however, even during the later phase a reconnection of a single vortex at the cylindrical wall, while a Kelvin wave expands along this vortex, remains the dominant mechanism of vortex generation. This is seen from the fact that the curve for $N$ follows closely that of the successful surface reconnections (dashed curve marked as "+1"). In comparison such surface reconnections are few in which a small loop is created, but which later shrinks away, for instance, because it is adversely oriented with respect to the azimuthal cf (dashdotted "removed" curve [which actually includes such small loops from both the surface and the bulk]). The dashed curve denoted as "0" refers to processes where a closed vortex ring from the bulk drifts against the cylindrical wall. Such cases do not change 


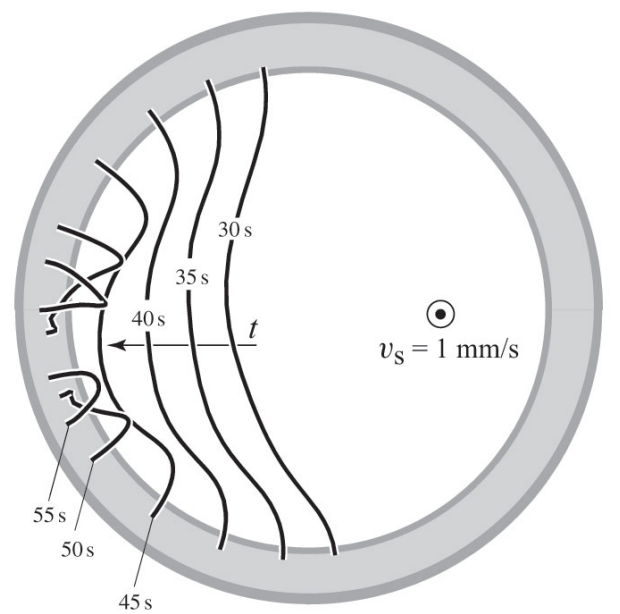

Fig. 17 (Color online) Boundary-mediated vortex formation in pipe flow. The calculation shows how a seed vortex travels across the cross section of the pipe after its release. The perspective view looks straight into the pipe against the flow, the inner ring is at $z=20 \mathrm{~mm}$ and the outer at $z=70 \mathrm{~mm}$. Originally at $t=0$ the center of the seed vortex was at $z=0$ closer to the pipe wall on the right. The vortex drifts both along the pipe (towards the viewer) and across the flow channel (from right to left). Its center section adopts the curvature of the pipe and annihilates (at $45<t<50 \mathrm{~s}$ ). The small loops at both ends survive as independent vortices, they are formed to satisfy the boundary condition. Reconnection kinks appear on both loops (at $t=50 \mathrm{~s}$ ), but these do not expand. The two loops reorient themselves with respect to the flow and then drift across the flow channel in the opposite direction. In this way the number of vortices has increased by one. The repolarization of the two new loops happens within the time span $50-55 \mathrm{~s}$, as seen in Fig. 18 The present figure shows the start of the calculation in Fig. 19 and thus the parameters are here, as well as in Fig. 18 the same as in Fig. 19

the value of $N$. They require successful vortex-generating reconnections in the bulk and consequently the dashed "0" curve emerges only after the solid "+1" curve has acquired sufficient slope. In contrast the solid " 0 " curve represents reconnections in the bulk between two different vortices which after the first $40 \mathrm{~s}$ rapidly becomes the most frequent event. These inter-vortex reconnections do not lead to changes in $N$ and are primarily associated with processes occurring between the twisted vortices within the bundle, where they help to increase the polarization of the vortices along the rotation axis. One might ask whether such bulk reconnections nevertheless emit Kelvin wave excitations which then propagate to the boundary and lead to loop formation and reconnections there. At present there is no clear evidence of that.

The insert in Fig. 15 compares the rates of vortex generation from reconnections at the wall and in the bulk. The dominant role of wall reconnections is compelling. Other similar calculations lead to the same conclusion: The reconnection of a single vortex at the cylindrical wall is the most important mechanism for the generation of new vortices. This process was illustrated by means of a numerical example in Ref. 4, while the task of Fig. 15 is to provide quantitative estimates of the relative frequencies of successful vortex-generating reconnections at the wall and in the bulk.

The second important consideration is correspondence with measurement. The obvious difference between calculation and measurement is the ease with which new vortices are generated in experiment below $T_{\text {on }}$, whereas in Fig. 15 the rate of vortex 


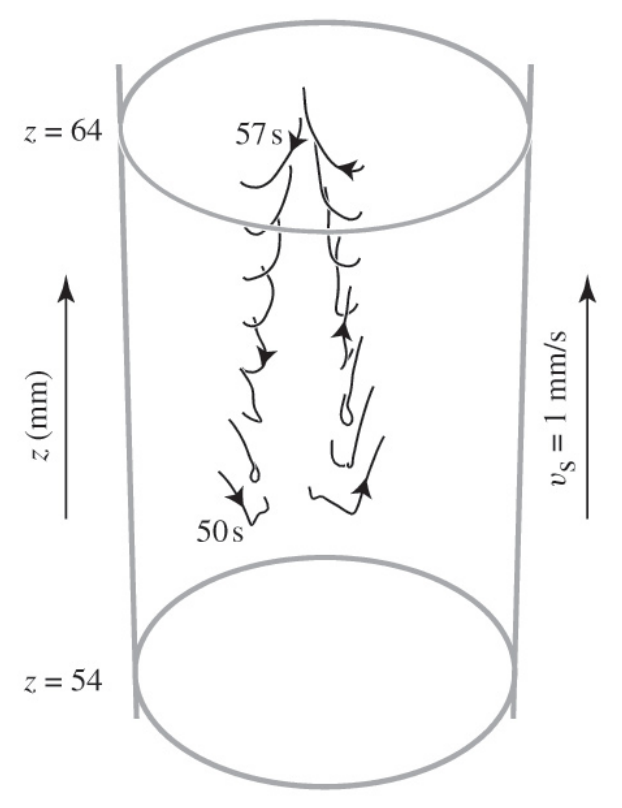

Fig. 18 (Color online) Reorientation of the two new vortex loops in Fig. 17 shown at $1 \mathrm{~s}$ intervals. On the bottom $(t=50 \mathrm{~s})$ the two loops have just formed on the back wall of the pipe and have the wrong orientation with respect to flow, while at the top $(t=57 \mathrm{~s})$ they have changed their orientation and start expanding towards the viewer. The arrows on the loops show the orientation of the circulation vector $\kappa$. The parameters are the same as in Fig. 19

generation remains modest. No clearly identifiable turbulent burst can be distinguished in Fig. 15. The same calculation at a lower rotation velocity of $0.8 \mathrm{rad} / \mathrm{s}$ gives a qualitatively similar result, both with respect to $\dot{N}(t)$ and the break down in different types of reconnections, except that all rates are smaller. After about $150 \mathrm{~s}$ both the surface and bulk rates turn off simultaneously and vortex generation stops at $N \approx 290$ vortices, well below the equilibrium number $N_{\text {eq }} \approx 570$. Clearly in this example no turbulent burst takes place, which would boost the vortex number up to $N_{\text {eq. }}$. In Fig. 15 at $0.9 \mathrm{rad} / \mathrm{s}$ the calculation has been continued to $115 \mathrm{~s}$ and $N \approx 400$ vortices, where vortex generation starts to slow down, again well short of $N_{\text {eq }} \approx 650$ vortices. The calculations are time consuming which limits our possibilities to obtain a more comprehensive understanding of their predictions.

It thus appears as if some mechanism is missing from the calculations in comparison to experiment, which makes vortices more unstable and adds to the vortex generation rate. The difference is less likely to reside in the bulk than on the cylindrical wall, where the condition of an ideal solid boundary should be examined closer. Nevertheless, at low vortex density Kelvin-wave formation on a single vortex followed by a reconnection at the surface is the only efficient mechanism for generating a new vortex. The problem is complex, since Kelvin-wave formation depends on the flow velocity and the orientation of the flow with respect to the vortex, which in turn change continuously while the vortex moves and the Kelvin wave itself starts to propagate. It appears that with respect to the reconnection of Kelvin-wave loops at the wall, the rotating cylinder with circular cross section is a particularly stable flow geometry. The next example calculates the equivalent of Fig. 15 for linear flow in a circular pipe. Here vortices turn out to be 


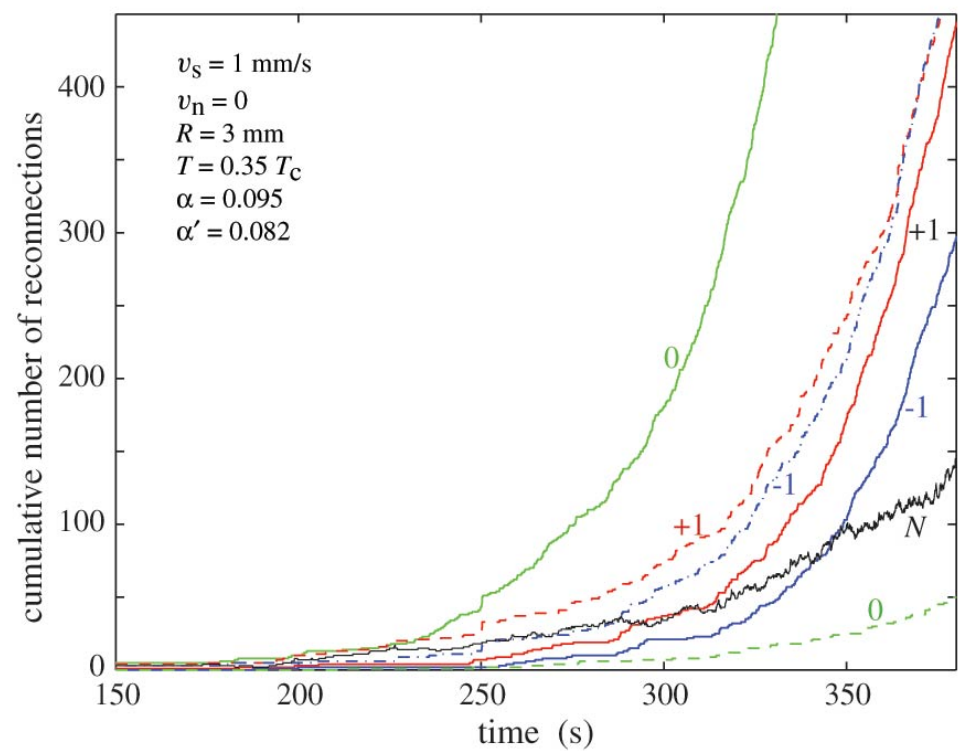

Fig. 19 (Color online) Calculated build up in the cumulative number of vortices and reconnections in linear pipe flow with circular cross section: (0, solid curve) reconnections in the bulk which do not change $N,(+1$, dashed) reconnections at the boundary which add one new vortex loop, (-1, dash-dotted) very small contracting loops both on the surface and in the bulk which approach the resolution limit and are removed, $(+1$, solid) reconnections in the bulk which add one vortex, $(-1$, solid) reconnections in the bulk between a closed loop and another vortex which remove one vortex, $(\mathrm{N})$ total number of vortices, and (0, dashed) reconnections at the boundary which do not change $N$.

less stable than in rotating flow. The reason is the enhanced role of reconnections at the wall.

Vortex generation in pipe flow:-Technically a measurement with seed-vortex injection in vortex-free linear pipe flow of superfluid ${ }^{3} \mathrm{He}-\mathrm{B}$ is a demanding task; so far such measurements have not been performed. Nevertheless, we present here calculations on a circular straight tube which is initially vortex-free. The calculations are performed similar to those above on rotating flow, but by approximating the boundary conditions with the faster image vortex techniques. The $\mathrm{cf}$ is enforced by imposing on the superfluid component flow at constant velocity over the cross section of the pipe.

Technically such measurements could be set up in the following manner: Suppose that both the entrance and the exit of the tube are covered with a superleak which prevents the flow of the normal component. The superfluid component is forced into motion with a piston acting on a large reservoir in front of the superleak-covered entrance of the flow tube. Obviously in a real experiment of this kind large numbers of vortices would be created in the superleak. These would continuously flood the tube, as long as the flow at constant mass rate is maintained. Such a measurement would not be informative about vortex generation and the onset of turbulence. To avoid this problem, the tube could be bent to a closed ring, in which the flow is created by rotating, in the same way as in the rotating cylinder.

In our numerical calculation we assume ideal laminar flow through the circular straight tube. To start vortex generation, we place one straight vortex line in the flow, 

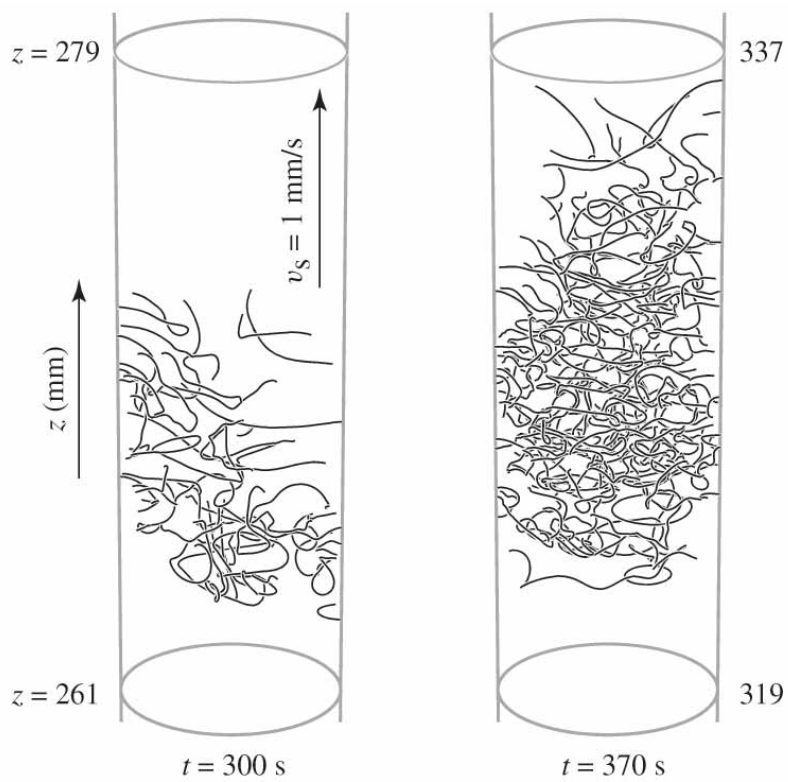

Fig. 20 Two snapshots from the evolution of the vortex tangle in pipe flow in Fig. 19 On the left the tangle is shown $300 \mathrm{~s}$ after the start from one seed vortex and on the right after $370 \mathrm{~s}$. During their evolution the vortices have drifted in the pipe along the $z$-axis (dimensions are given in $\mathrm{mm}$ ), where $z=0$ was fixed at the middle point of the seed vortex at $t=0$. The parameters are the same as in Fig. 19

which stretches from wall to wall across the tube, slightly tilted from the perpendicular plane, to break the symmetry. It turns out that the later evolution of the seed vortex is rather insensitive to its initial configuration and that our results do not depend materially on how the seed vortex was originally placed in the tube. Experimentally seed vortex injection can be achieved by creating vortex rings in applied flow by means of the neutron capture reaction in neutron irradiation $[22$.

In Fig. 17 we examine the trajectory of the seed vortex along and across the flow channel. A flat velocity distribution $v_{\mathrm{s}}=1 \mathrm{~mm} / \mathrm{s}$ is imposed on the superfluid component over the cross section (while the motion of the normal component is clamped by the superleaks or by a large kinematic viscosity, $v_{\mathrm{n}}=0$ ). The vortex drifts with roughly the velocity $v_{\text {s }}$ along the pipe downstream ( transverse across the tube, driven by the dissipative mutual friction force $\propto \alpha v_{\mathrm{s}}$. The consecutive configurations of the vortex are shown in Fig. 17 at $5 \mathrm{~s}$ intervals. Owing to the boundary condition on the wall, the vortex bows out in the center and mimics the curvature of the circular pipe wall, while it traverses across the entire cross section. Ultimately its center section, which is now aligned along the pipe wall, annihilates. Only one small loop from both ends of the original vortex remains, the vestiges from the boundary condition. In this example both of these end loops manage to reorient themselves with respect to the flow direction (Fig. 18) and then start an expanding motion in the opposite direction across the flow. A successful reorientation is not always the case; often one end loop may simply contract and annihilate. However, in Fig. 18 the number of vortices starts to grow continuously from one single seed vortex. Here the transverse flight time across the flow is approximately $60 \mathrm{~s}\left(\sim 2 R /\left(\alpha v_{\mathrm{s}}\right)\right.$. Thus 


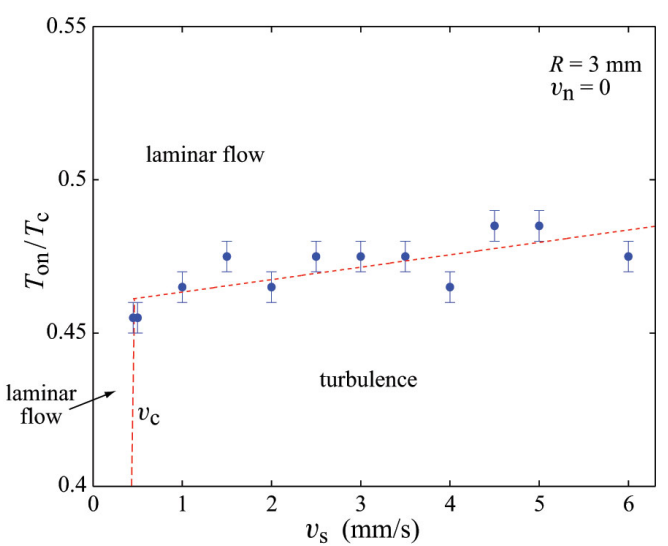

Fig. 21 (Color online) Phase diagram of flow states and dependence of the onset temperature of turbulence on flow velocity in linear pipe flow. The calculations are started with a single initial vortex, as in Fig. 19 At low velocities below the almost vertical critical boundary sustained dynamic vortex generation cannot be maintained at any temperature down to below $0.3 T_{\mathrm{c}}$ and a state of turbulent flow is not created. At higher velocities above this critical boundary sustained turbulence exists at and below the onset temperature plotted on the vertical scale. The parameters are the same as in Fig. 19

after the first $60 \mathrm{~s}$ we have two vortices, four vortices after $\sim 120 \mathrm{~s}$, and after $\sim 180 \mathrm{~s}$ $N$ starts increasing more rapidly, as seen in Fig. 19

Comparing Figs. 15 and 19 it is evident that for pipe flow - in contrast to rotating flow - there is no difficulty in starting turbulence from a single seed vortex in the simulation calculation. The reason is the difference in flow geometry: As seen in Fig. 17 for pipe flow the generation of new vortices does not depend only on the successful expansion of Kelvin-waves, but is aided by the boundary condition which gives rise to characteristic end loops. Nevertheless, in both calculations it is the interaction with the wall which is responsible for the early phase of vortex generation, in Fig. 19 up to about $300 \mathrm{~s}$. After about $360 \mathrm{~s}$ the generation and annihilation of loops in surface reconnections compensate each other and from here onwards the generation in bulk becomes mainly responsible for the production of new vortices.

Interestingly, also in Fig. 19 reconnections between two different vortices in the bulk, which do not directly lead to new vortices, soon dominate over all other processes. These bulk reconnections abound, when a sufficiently dense tangle has formed and vortices traverse across the flow in both directions, as seen in the two snapshots in Fig. 20. This leads to rapidly changing configurations in the tangle. Furthermore, since all vortices travel downstream, a turbulent plug is formed which hardly if at all spreads upstream (pinning is excluded from our numerical model). Ultimately, when the vortex plug reaches the superleak at the exit of the flow tube, the vortices are annihilated and the original state of vortex-free flow reappears.

It is instructive to note that turbulent plugs have been observed in the flow of superfluid ${ }^{4} \mathrm{He}$ along a straight capillary tube with circular cross section 33 . In these measurements temperature fluctuations are registered along the tube in steady state flow conditions in some velocity range. The fluctuations are interpreted to arise from the flow of turbulent plugs along the tube. The plugs consist of a tangle of quantized vortices, they extend over a limited length of the tube, and display relatively sharp fronts with the laminar flow sections. However, presumably in the case of ${ }^{4} \mathrm{He}-\mathrm{II}$ the 


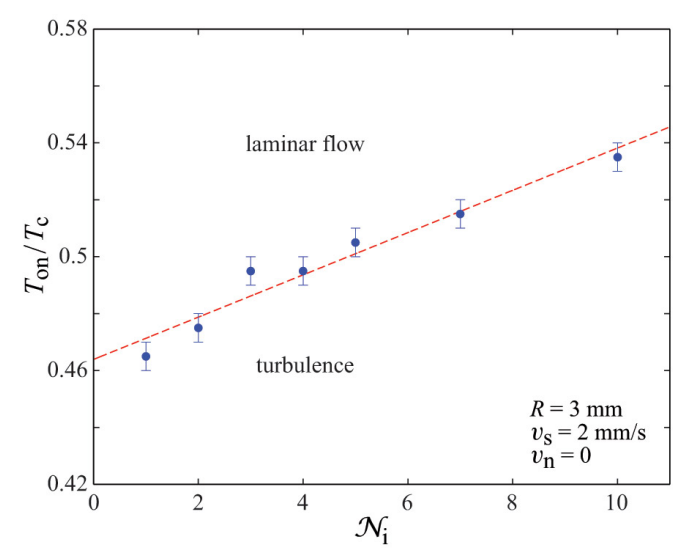

Fig. 22 (Color online) Dependence of the onset temperature of turbulence on the number of seed vortices in linear pipe flow. The calculations are started with $\mathcal{N}_{\mathrm{i}}$ seed vortices which are placed transverse across the pipe at equidistant separation from each other, with their center points in the same cross section of the tube at $z=0$. Other conditions are the same as in Fig. 19 except for $v_{\mathrm{s}}=2 \mathrm{~mm} / \mathrm{s}$. At larger values of $\mathcal{N}_{\mathrm{i}}$ (above 15 seed vortices) the dependence saturates at about $T_{\mathrm{on}} / T_{\mathrm{c}} \leq 0.55$.

transition from laminar to turbulent flow is not governed by the supply of vortices, as discussed here for pipe flow of ${ }^{3} \mathrm{He}-\mathrm{B}$, but the formation of the plugs is regulated by the associated fluctuations in flow velocities, similar to the formation and decay of turbulent plugs in viscous pipe flow 34] (where the plugs are formed from classical eddies).

In Figs. 21 and 22 the calculations from Fig. 19 are carried further, to check for a dependence of the onset temperature of turbulence on the flow velocity $v_{\mathrm{s}}$ and on the number of seed vortices $\mathcal{N}_{\mathrm{i}}$. In Fig. 21 an almost linear dependence is found for $T_{\mathrm{on}}$, as a function of the velocity $v_{\mathrm{S}}$ which is imposed on the superfluid component. This line represents the onset temperature of the single-vortex instability: At and below this critical temperature a state of sustained turbulence is observed to develop. At very low velocities $\left(v_{\mathrm{s}} \lesssim 0.4 \mathrm{~mm} / \mathrm{s}\right)$ a region is found where no vortex instability and no dynamic generation of new vortices occurs at any temperature down to below $0.3 T_{\mathrm{c}}$. This almost vertical boundary we associate with the critical velocity of turbulence at vanishingly small mutual friction. In Fig. 22 in turn, we see that the onset temperature depends on the initial number of seed vortices with which the calculation is started: A roughly linear dependence $T_{\mathrm{on}}\left(\mathcal{N}_{\mathrm{i}}\right)$ is found. Figs. 21 and 22 are thus both consistent with what we expect on the basis of the rotating experiments (compare to Figs. 8 and 10. In our calculations on rotating flow these dependences have not emerged with equal clarity, owing to the higher stability of evolving vortices in the rotating cylinder. In contrast pipe-flow calculations, performed with the same conventions and approximations, straightforwardly lead to the expected relations.

\section{SUMMARY}

A quantized vortex is a topologically stable structure of the superfluid order parameter field. In principle, it should be possible to account for the appearance of every new vortex. In ${ }^{4} \mathrm{He}-\mathrm{II}$ this has notoriously been a difficult task: Vortices appear to emerge out 
of nowhere, without apparent systematics. Various mechanisms have been proposed to explain their origin. In ${ }^{3} \mathrm{He}-\mathrm{B}$ at temperatures below $2.3 \mathrm{mK}$ vortex formation processes are instabilities and not thermally activated. Vortex formation is here in better control, partly owing to the two or three orders of magnitude larger vortex core diameter and the reduced influence from surface roughness. Moreover, vortex formation can here be examined as a function of a mutual friction dissipation which has a strong, almost exponential temperature dependence around the critical regime $\zeta \sim 1$.

With decreasing mutual friction the stability of vortices is reduced and at $T \sim 0.6 T_{\mathrm{c}}$ turbulence in the bulk volume becomes possible. This hydrodynamic transition from laminar to turbulent dynamics takes place at a somewhat higher temperature than where an isolated evolving vortex might become unstable in the rotating column. Before turbulence can be started from single vortices evolving at low density, new expanding loops have to be generated by a precursor mechanism. This happens via the singlevortex instability for which the probability rapidly increases with reducing friction. Thus the cascade process, the single-vortex instability followed by a localized turbulent burst in the bulk volume, becomes possible. Experimentally this is observed as an abrupt change in the stability of the dynamics, manifested as a sudden transition to turbulence within a narrow temperature interval. The simplest means to investigate the instability is to measure at constant rotation velocity the onset temperature $T_{\text {on }}$ of turbulence after the introduction of a controlled number of seed vortices. Examples of such measurements are shown in Figs. 3 and 14 . where $T_{\text {on }}$ has been determined in each of the four graphs. In Sec. 2 we have analyzed individual data points in such graphs, in order to characterize the properties of the single-vortex instability. It thereby turns out that in the onset temperature regime, $T \approx T_{\text {on }}$, a fraction of the transitions to turbulence display prolonged precursory vortex formation at slow rate, before the turbulent burst in the bulk sets in. At temperatures further below the onset regime the instabilities proceed too rapidly to be captured with our measuring techniques.

Our numerical simulation calculations in Sec. 3 show that interactions of the evolving seed vortex with the cylindrical wall in the presence of rotating counterflow is the predominant source for new vortices in the low density regime, before interactions between vortices in the bulk become possible. In these calculations the walls are represented with the boundary conditions of an ideal solid surface. The experimental results suggest, however, that surface properties or geometrical features do influence the onset temperature, as seen in Fig. 13. More realistic boundary conditions might therefore be needed and might reduce the main disagreement between the rotating experiments and present calculations, namely enhance the probability of wall reconnections and the formation of new expanding loops in the calculations. In contrast, our calculations on flow in a straight circular pipe suggest that in this geometry wall interactions lead to the generation of new vortices at higher temperatures and at lower flow velocities than in rotation. Of these two types of flow, rotation, which promotes polarization of vortices along the rotation axis, is a more stable environment for dynamically evolving vortices. This is in particular the case for a long smooth-walled circular column with good alignment along the rotation axis. The aligned circular column appears to be a most stable special case [8] compared to, for instance, a column which is inclined by a large amount with respect to the rotation axis or one with square cross section 35. Similar differences in stability are known to apply for viscous pipe flow with circular versus square cross section of the flow channel 34 .

Finally we note that the single-vortex instability starts in the rotating column a sequence of events, which have been described in a recent review [8]. Of central interest 
here is the propagation of polarized vortices along the rotating circular column and how this is changed by the increasing turbulent influence with decreasing temperature [28. These phenomena elucidate superfluid turbulence in the $T \rightarrow 0$ limit, which recently has been studied also in two other types of measurements, by monitoring the decay of homogeneous isotropic turbulence, after the external pumping is switched off [35, 36].

Acknowledgments:- This work is supported by the Academy of Finland (grants 213496, 124616, and 114887) and by ULTI research visits (EU Transnational Access Programme FP6, contract RITA-CT-2003-505313).

\section{References}

1. A.P. Finne, T. Araki, R. Blaauwgeers, V.B. Eltsov, N.B. Kopnin, M. Krusius, L. Skrbek, M. Tsubota, and G.E. Volovik, Nature 424, 1022 (2003)

2. A.P. Finne, V.B. Eltsov, R. Hänninen, N.B. Kopnin, J. Kopu, M. Krusius, M. Tsubota, and G.E. Volovik, Rep. Prog. Phys. 69, 3157 (2006)

3. V.M.H. Ruutu, Ü. Parts, J.H. Koivuniemi, N.B. Kopnin, and M. Krusius, J. Low Temp. Phys. 107, 93 (1997); Ü. Parts et al., Europhys. Lett. 31, 449 (1995)

4. A.P. Finne, V.B. Eltsov, R. Hänninen, J. Kopu, M. Krusius, E.V. Thuneberg, and M. Tsubota, Phys. Rev. Lett. 96, 85301 (2006)

5. E.L. Andronikashvili and Yu.G. Mamaladze, Prog. Low Temp. Phys. Vol. V, p. 79, ed. C.J. Gorter (North-Holland Publ., Amsterdam, 1967)

6. W.F. Vinen and J. Niemela, J. Low Temp. Phys. 128, 167 (2002); W.F. Vinen, J. Low Temp. Phys. 145, 7 (2006)

7. H.P. Greenspan, The theory of rotating fluids (Cambridge University Press, Cambridge, UK, 1968)

8. V.B. Eltsov, R. de Graaf, R. Hänninen, M. Krusius, R.E. Solntsev, V.S. Lvov, A.I. Golov, P.M. Walmsley, Prog. Low Temp. Phys. Vol XVI, ed. M. Tsubota (Elsevier B.V., Amsterdam, 2008); preprint - arXiv:0803.3225v2

9. R. Hänninen, A. Mitani, and M. Tsubota, J. Low Temp. Phys. 138, 589 (2005)

10. R.E. Solntsev, R. de Graaf, V.B. Eltsov, R. Hänninen, and M. Krusius, J. Low Temp. Phys. 148, 311 (2007)

11. R. Hänninen, V.B. Eltsov, A.P. Finne, R. de Graaf, J. Kopu, M. Krusius, and R.E. Solntsev, preprint - arXiv:cond-mat/0701647 (2007)

12. V.B. Eltsov, A.P. Finne, R. Hänninen, J. Kopu, M. Krusius, M. Tsubota, and E.V. Thuneberg, Phys. Rev. Lett. 96, 215302 (2006); J. Low Temp. Phys. 150, $373(2008)$

13. T.D.C. Bevan, A.J. Manninen, J.B. Cook, A.J. Armstrong, J.R. Hook, and H.E. Hall, J. Low Temp. Phys. 109, 423 (1997); Phys. Rev. Lett. 74, 750 (1995)

14. K.W. Schwarz, Phys. Rev. Lett. 64, 1130 (1990); ibid. 69, 3342 (1992)

15. V.M. Ruutu, J.J. Ruohio, M. Krusius, B. Plaçais, and E.B. Sonin, Physica B 255, $27(1998)$

16. A.P. Finne, R. Blaauwgeers, S. Boldarev, V.B. Eltsov, J. Kopu, and M. Krusius, AIP Conf. Proc. 850, 177 (2006)

17. R. Blaauwgeers, V.B. Eltsov, G. Eska, A.P. Finne, R.P. Haley, M. Krusius, J.J. Ruohio, L. Skrbek, and G.E. Volovik, Phys. Rev. Lett. 89, 155301 (2002)

18. A.P. Finne, S. Boldarev, V.B. Eltsov, and M. Krusius, J. Low Temp. Phys. 136, $249(2004)$

19. A.P. Finne, S. Boldarev, V.B. Eltsov, and M. Krusius, J. Low Temp. Phys. 138, $567(2005)$ 
20. V.B. Eltsov, M. Krusius, and G.E. Volovik, Prog. Low Temp. Phys. Vol. XV, p. 1, ed. W.P. Halperin (Elsevier B.V., Amsterdam, 2005)

21. T.W.B. Kibble, in Patterns of Symmetry Breaking, p. 3, eds. H. Arodz, J. Dziarmaga, and W.H. Zurek, NATO Science Series in Mathematics, Physics, and Chemistry, Vol. 127 (Kluwer Academic Publishers, Dordrecht, 2003); W.H. Zurek, Phys. Rep. 276, 177 (1996)

22. A.P. Finne, S. Boldarev, V.B. Eltsov, and M. Krusius, J. Low Temp. Phys. 135, $479(2004)$

23. P.J. Hakonen, M. Krusius, M.M. Salomaa, R.H. Salmelin, J.T. Simola, A.D. Gongadze, G.E. Vachnadze, and G.A. Kharadze, J. Low Temp. Phys. 76, 225 (1989)

24. A.I. Ahonen, M.A. Paalanen, and M. Krusius, J. Low Temp. Phys. 25, 421 (1976)

25. M. Blăzková, M. Človečko, V.B. Eltsov, E. Gazo, R. de Graaf, J.J. Hosio, M. Krusius, D. Schmoranzer, W. Schoepe, L. Skrbek, P. Skyba, R.E. Solntsev, and W.F. Vinen, J. Low Temp. Phys. 150, 525 (2008)

26. J. Kopu, R. Schanen, R. Blaauwgeers, V.B. Eltsov, M. Krusius, J.J. Ruohio, and E.V. Thuneberg, J. Low Temp. Phys. 120, 213 (2000)

27. A.P. Finne, V.B. Eltsov, R. Blaauwgeers, Z. Janu, M. Krusius, and L. Skrbek, J. Low Temp. Phys. 134, 375 (2004)

28. V.B. Eltsov, A.I. Golov, R. de Graaf, R. Hänninen, M. Krusius, V. L'vov, and R.E. Solntsev, Phys. Rev. Lett. 99, 265301 (2007)

29. L. Skrbek, R. Blaauwgeers, V.B. Eltsov, A.P. Finne, N.B. Kopnin, and M. Krusius, Physica B 329-333, Pt. 1, 106 (2003)

30. K.W. Schwarz, Physica B 197, 324 (1994)

31. K.W. Schwarz, Phys. Rev. B 38, 2398 (1988) and references there

32. K.W. Schwarz, Phys. Rev. B 31, 5782 (1985)

33. H. van Beelen, W. van Joolingen, and K. Yamada, Physica B 153, 248 (1988); G. Marees, R.F. Mudde, and H. van Beelen, Physica B 144, 292 (1987)

34. T. Mullin and J. Peixinho, J. Low Temp. Phys. 145, 75 (2006); Phys. Rev. Lett. 96, 094501 (2006); B. Hof, A. Juel, and T. Mullin, Phys. Rev. Lett. 91, 244502 (2003)

35. P.M. Walmsley, A.I. Golov, H.E. Hall, A.A. Levchenko, and W.F. Vinen, 2007, Phys. Rev. Lett. 99, 265302 (2007); P.M. Walmsley and A.I. Golov, Phys. Rev. Lett. 100, 245301 (2008)

36. S.N. Fisher, A.J. Hale, A.M. Guénault, and G.R. Pickett, Phys. Rev. Lett. 86, 244 (2001); D.I. Bradley, D.O. Clubb, S.N. Fisher, A.M. Guénault, R.P. Haley, C.J. Matthews, G.R. Pickett, V. Tsepelin, and K. Zaki, Phys. Rev. Lett. 96, 35301 (2006); D.I. Bradley, S.N. Fisher, A.M. Guénault, R.P. Haley, S. O Sullivan, G.R. Pickett, and V. Tsepelin, Phys. Rev. Lett. 101, 065302 (2008) 